\title{
A CAPILLARY SURFACE WITH NO RADIAL LIMITS
}

\author{
A Dissertation by \\ Colm Patric Mitchell \\ Master of Science, Wichita State University, 2009 \\ Bachelor of Science, Wichita State University, 2005
}

Submitted to the Department of Mathematics and the faculty of the Graduate School of Wichita State University in partial fulfillment of the requirements for the degree of Doctor of Philosophy 
(C)Copyright 2017 by Colm Patric Mitchell

All Rights Reserved 


\section{A CAPILLARY SURFACE WITH NO RADIAL LIMITS}

The following faculty members have examined the final copy of this dissertation for form and content, and recommend that it be accepted in partial fulfillment of the requirement for the degree of Doctor of Philosophy with a major in Applied Mathematics.

Thomas DeLillo, Committee Chair

James Steck, Committee Member

Elizabeth Behrman, Committee Member

Ziqi Sun, Committee Member

$\overline{\text { Jason Ferguson, Committee Member }}$

Accepted for the College of Liberal Arts and Sciences

Ron Matson, Dean

Accepted for the Graduate School

Dennis Livesay, Dean 


\section{DEDICATION}

To my wife Jenny, without her gentle shove I may never have gone to college; and to my boys Caleb and Connor, who had many years of dad time taken up in my studies. 


\section{ACKNOWLEDGEMENTS}

I wish to acknowledge the tireless efforts of Dr. Kirk Lancaster over the last several years. Without his guidance I may never have finished my dissertation. It is unfortunate that he could not be present to chair the committee at the defense.

I wish to thank Dr. Thomas DeLillo for stepping in for Kirk to chair the committee at the last minute. 


\begin{abstract}
We begin by discussing the circumstances of capillary surfaces in regions with a corner, both concave and convex. These circumstances led to the (since proved) Concus-Finn Conjecture, which gives the requirements for a continuous solution to the capillary problem. We include the requirements for the existence of radial limits and fans of radial limits in these corner regions. We outline the method for which conformal mapping of the Gauss map of these surfaces can be computed to allow the analytic extension of many related theorems. Finally, we introduce a broadening of the existing research by taking an example done with Dirichlet conditions in 1996 by Kirk Lancaster and David Siegel and recreating the example with contact angle data instead. With the use of contact angle data we find that where in the original example $\gamma$ could not be bounded away from zero or $\pi$, we were able to find a capillary surface such that $\gamma$ is bounded away from 0 and $\pi$.
\end{abstract}




\section{TABLE OF CONTENTS}

1 The Capillary Problem in a Cylinder . . . . . . . . . . . . . . . . 1

$1.1 \quad$ Organization . . . . . . . . . . . . . . . . . . 1

1.2 A Bit of Background . . . . . . . . . . . . . . . . . . . 1

1.3 Recent Research Efforts . . . . . . . . . . . . . . . . . 3

1.4 The Capillary Surface in a Cylinder . . . . . . . . . . . . . . . . 5

1.5 Capillary surfaces near Corners . . . . . . . . . . . . . . 6

1.6 The Concus-Finn Conjecture . . . . . . . . . . . . . . . . . 7

1.7 Radial Limits, Central Fans and Continuity at Corners . . . . . . . . . 11

1.7.1 Convex Corners . . . . . . . . . . . . . . . . 14

1.7.2 Nonconvex Corners . . . . . . . . . . . . . . . . . . . 15

1.8 Constructing a Capillary Graph . . . . . . . . . . . . . . 17

2 Riemann-Hilbert and the Scheme of Things . . . . . . . . . . . . 23

2.1 Solving the Riemann-Hilbert Problem . . . . . . . . . . . . . . . . . 23

2.2 Computing A Capillary Surface . . . . . . . . . . . . . . . 30

2.3 Conformal Mapping . . . . . . . . . . . . . . . . . . 32

3 A Capillary Surface with No Radial Limits . . . . . . . . . . . . . . 36

3.1 Introduction . . . . . . . . . . . . . . . . 36

3.2 A Surface Defined by Contact Angle Data . . . . . . . . . . . . 38

3.3 Concluding Remarks . . . . . . . . . . . . . . . . . . 42

REFERENCES . . . . . . . . . . . . . . . . . . . . . . . 43 


\section{Chapter 1}

\section{The Capillary Problem in a Cylinder}

\section{$1.1 \quad$ Organization}

Recalling my Master's Thesis [42], we begin with chapter 1 by discussing the circumstances of capillary surfaces in regions with a corner, both concave and convex. These circumstances led to the (since proved) Concus-Finn Conjecture, which gives the requirements for a continuous solution to the capillary problem. We include the requirements for the existence of radial limits and fans of radial limits in these corner regions. As with my Master's Thesis, in chapter 2 we outline the method for which conformal mapping of the Gauss map of these surfaces can be computed to allow the analytic extension of many related theorems. The ideas presented in these first two chapters are the source of the computations in Francis Nguyen's Master's Thesis [47]. Finally, in chapter 3 we introduce a broadening of the existing research by taking an example done with Dirichlet conditions in 1996 by Kirk Lancaster and David Siegel and recreating the example with contact angle data instead. With the use of contact angle data we find that where in the original example $\gamma$ could not be bounded away from zero or $\pi$, we were able to find a capillary surface such that $\gamma$ is

bounded away from 0 and $\pi$. [10]. We conclude with an extension of the example in [37] of a region of no radial limits with Dirichlet Data to a region of no radial limits with Contact Angle Data which has been submitted for publication [43].

\subsection{A Bit of Background}

In the early $18^{\text {th }}$ century, observations of capillarity phenomena were published in the Philosophical Transactions of the Royal Society. In 1710, Francis Hauksbee studied capillary surfaces in wedge domains and published several observations of those capillary wedge domains in the Philosophical Transactions. Brook Taylor had articles such as Part of a Letter from Mr. Brook Taylor, F. R. S. to Dr. Hans Sloane R. S. Secr. Concerning the 
Ascent of Water between Two Glass Planes (1710), which involved observations of capillarity phenomena, published in the same journal.

The topic which we now call "curvature" was discussed during the $18^{\text {th }}$ century but the formulation of the notion of "mean curvature" was developed by Thomas Young in 1805 and Pierre-Simon Laplace in 1806; they observed that the "mean curvature" of the free surface should be proportional to the change in pressure across the surface and that the fluid should meet the boundary walls with a constant angle. The derivations of Young and Laplace were not rigorous; Carl Friedrich Gauss rigorously proved the "Young-Laplace equation" and the "contact angle boundary condition" in 1830.

In the second half of the $19^{\text {th }}$ century, the Belgian physical chemist J.A.F. Plateau studied soap films (i.e. minimal surfaces), soap bubbles (i.e. constant mean curvature surfaces), capillary surfaces, the surface tension of fluids and many other phenomena of importance to surface chemistry and other disciplines. By using fluids of equal density, he was able to study surfaces for which the effects of gravity were essentially absent. Plateau and later Lord Rayleigh studied the stability of liquid columns. Common numerical procedures were developed for and tested on capillary problems such as hanging drops during the $19^{\text {th }}$ century.

Capillarity fell out of favor in the first half of the 20th century and little significant progress was recorded. Interest in capillarity phenomena underwent a renewal in the second half of the twentieth century, in part because of interest in space exploration and of advances in chemical engineering and surface chemistry. The modern mathematical theory of capillarity developed after 1950. During the decades of 1950-1979, individuals like Paul Concus, Robert Finn, Ennio Di Giorgi, Mario Miranda, M. Emmer, Herbert Federer, Fred Almgren, Jean Taylor, Enrico Giusti and others made significant contributions to this branch of the theory of calculus of variations. Since 1980, these researchers and many others have contributed to an explosion of knowledge about geometric analysis, geometric partial differential equations and the portion of the calculus of variations involved with "curvature problems." 


\section{$1.3 \quad$ Recent Research Efforts}

If the boundary $\partial \Omega$ of the domain $\Omega$ has a corner, then the cylindrical container $\Omega \times \mathbb{R}$ is a "wedge" and the behavior of capillary surfaces in such containers is of long-standing interest. In general, when the opening angle of the wedge is small when compared to the boundary data, bounded solutions of the capillary problem do not exist; several researchers such as Robert Finn, L.F.Tam, Erich Miersemann and others have investigated this behavior.

In other cases, we know the capillary problem has a bounded solution $u \in C^{2}(\Omega)$, but this solution might not be continuous at the corner which lies on $\partial \Omega$. We will assume this corner is located at $O=(\mathbf{0}, \mathbf{0})$ and that the tangent rays to the two sides of $\partial \Omega$ near $O$, denoted in Lancaster's notation as $\partial^{+} \Omega$ and $\partial^{-} \Omega$, (and we will continue this type of notation) form an angle of magnitude $2 \alpha$ for some $\alpha \in[0, \pi]$. We will assume $\alpha \in(0, \pi)$. What follows is a discussion of some of the literature on bounded solutions of the capillary problem, focusing on the literature which has the greatest influence on this work.

Sufficient conditions for the existence of a solution $u \in C^{2}(\Omega) \cap C^{0}(\bar{\Omega})$ of the capillary problem in a locally Lipschitz domain with constant contact angle $\gamma$ were obtained by Emmer in 1973 ( $[15])$; this publication was the first to obtain existence of capillary surfaces for domains with corners. For non-convex corners, in which $\alpha \in(\pi / 2, \pi)$, Emmer's necessary condition was too restrictive and more appropriate conditions were obtained in 1977 by Finn and Gerhardt ( [19]). In 1980, Simon ( [53]) obtained nearly optimal sufficient conditions for the continuity and differentiability at a convex corner(i.e. $0<\alpha<\pi / 2$ ) of a solution of the capillary problem when the contact angle was Hölder continuous in a neighborhood of the corner. Also in 1980, in fact in the same issue of the journal (Pacific Journal of Math) as Simon, Korevaar ( [27]) proved that at a reentrant corner (i.e. $\alpha \in(\pi / 2, \pi)$ ) with constant contact angle, the solution of the capillary problem can be discontinuous at the corner, even though it is bounded (see also [28], [29].) In 1986, Tam ( [54]) extended Simon's result by investigating the borderline condition and obtained the continuity of capillary surfaces in these borderline cases. In 1986, Finn's book Equilibrium Capillary Surfaces was published; 
this book contains examples, techniques and theorems of great interest and use.

In 1985, Lancaster ( [30]) studied a related problem, which was the Dirichlet problem for the minimal surface equation, when $\partial \Omega$ had a reentrant corner and established the existence and behavior of the radial limits at $O$ of solutions of the minimal surface problem (see also [12].) This paper introduced techniques which would be used subsequently to investigate Dirichlet and contact angle boundary value problems for mean curvature equations (e.g. [13], [31], [32], [33], [37], [38], [35].) For minimal surfaces, the stereographic projection $g$ of the Gauss map $\vec{N}$ is analytic (or "conjugate-analytic") and this fact can be used to obtain "capillary graphs" (i.e. minimal surfaces $z=u(x, y)$ which satisfy contact angle boundary conditions). The Weierstrass $(f, g)$ representation of minimal surfaces yield capillary graphs if appropriate analytic functions $f$ can be obtained; such functions are solutions of RiemannHilbert problems; this technique was used in [14], [11] and [36]. Huff and McCuan ( [22]) used a related technique in 2006.

In 1969, Concus and Finn ( [3]) investigated capillary surfaces in wedges and revisited these surfaces in 1996 in $([7]),([17]),($ see also [9].) In 1996, an article by Lancaster and Siegel ( [37]) was published and received a Featured Review ( [18]) by Math Reviews; this paper proved that radial limits at $O$ of bounded capillary surfaces exist under very weak conditions and characterized the behavior of these radial limits. It is this paper by Lancaster and Siegel that originally published the example that I modify in my research.

The existence of a "central fan" of (constant) radial limits was first established in [30] for solutions of the Dirichlet problem for the minimal surface problem, in [13] for solutions of the Dirichlet problem for mean curvature problems and for capillary surfaces in [37]. In his review of [37], Finn ( [18]) noted that general sufficient conditions for the existence of central fans were not given in [37]. In [51], Shi and Finn broke the symmetry of an example in [37] and showed that the resulting capillary surface could not have a central fan (see chapter 4). In other words, the symmetry requirement is sharp. In [36], Athanassenas and Lancaster formally presented the central fan question to the mathematical community. At the moment, 


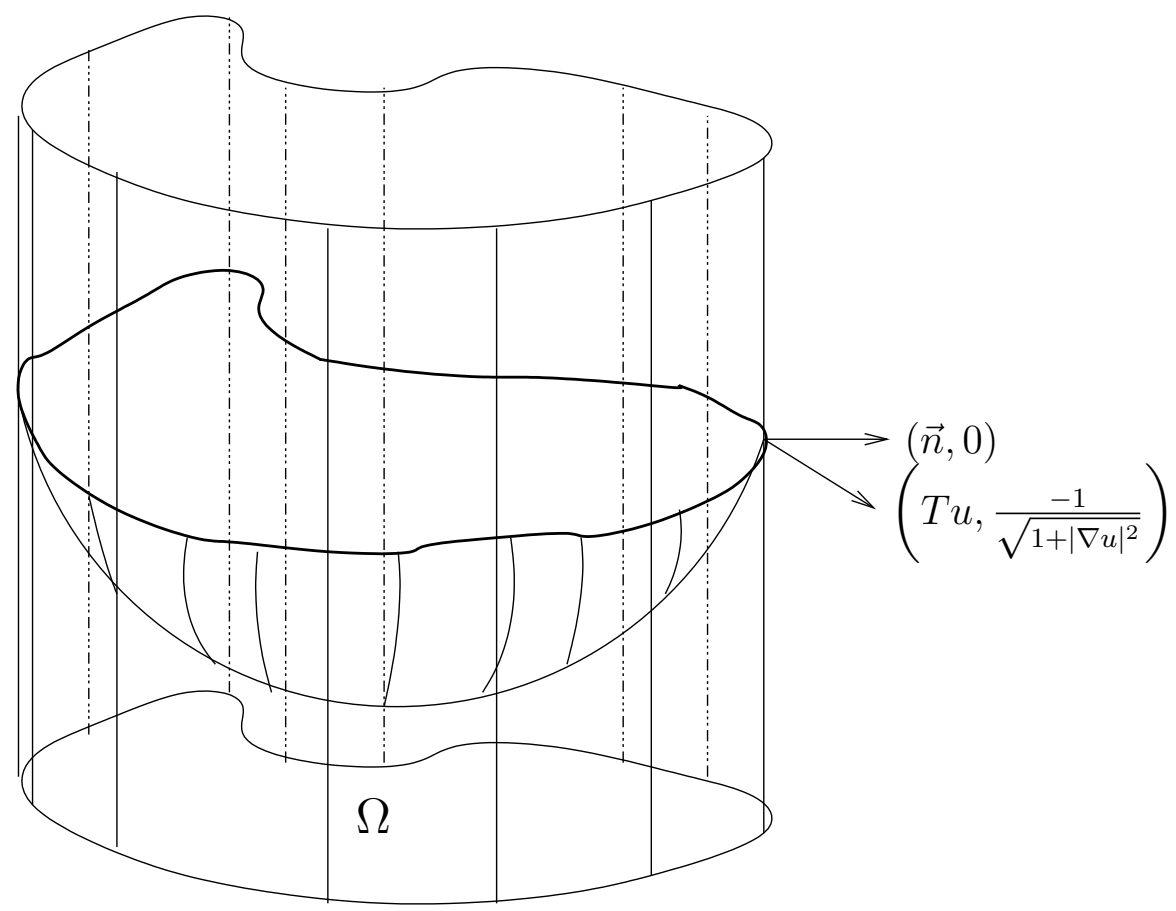

Figure 1.1: The Capillary Problem in a Cylinder

even conjectures about necessary and sufficient conditions for the existence of central fans are unknown.

\subsection{The Capillary Surface in a Cylinder}

A capillary surface is the interface separating two fluids, one of which could be a gas (e.g. air), that are adjacent to each other but not miscible. Alternately, a capillary surface is formed when one liquid meets a corner whose sides are made of differing materials, and thus the fluid has differing contact angles with each material resulting in some interesting behavior at the corner. Our attention will focus on a liquid partially filling a vertical cylinder $\Omega \times \mathbb{R}, \Omega \subset \mathbb{R}^{2}$ and the interface it makes with the sides of the cylinder at the corner $O$. It is this alternate definition that we investigate.

Our problem comes down to finding a function $f \in C^{2}(\Omega)$, that satisfies the boundary 
conditions in some way, such that

$$
\begin{aligned}
\operatorname{div} T f & =\kappa f+\lambda \text { in } \Omega \\
T f \cdot \nu & =\cos \gamma \text { on } \partial \Omega \\
T f & =\frac{\nabla f}{\sqrt{1+|\nabla f|^{2}}}
\end{aligned}
$$

where $\lambda$ is a Lagrange parameter arising from a volume constraint. Our constant $\kappa=\frac{\rho g}{\sigma}$ is the "capillary" constant; $\rho$ is the change in density across the surface, $g$ is gravity, and $\sigma$ is the surface tension; $\nu$ is the outward unit normal on $\partial \Omega$, and $\gamma$ is the contact angle made by the liquid on each type of surface and therefore is a function of position $(\gamma(s))$ on $\partial \Omega$ such that $0 \leq \gamma(s) \leq \pi$

The surface $z=f(x, y)$ describes the shape of the static liquid-gas interface in a vertical cylinder of cross-section $\Omega$. The cases $\kappa>0, \kappa=0, \kappa<0$ correspond to positive (e.g. sitting or sessile drops), zero, and negative (e.g. hanging or pendant drops) gravity, respectively. If the liquid, gas, and cylinder are each homogeneous, then $\gamma$ is constant. Finn [16] presents general existence theory that covers the case $\kappa \geq 0$ and provides existence of a bounded variational solution that may be shown to have more regularity for the $\kappa \geq 0$ case. Siegel provides existence for a classical solution in the $\kappa>0$ case under less restrictive hypotheses, although this solution may not be bounded. There is also existence theory for the $\kappa<0$ and $|\kappa|$ small cases. $([34])$

\subsection{Capillary surfaces near Corners}

As stated previously, we consider domains $\Omega \subset \mathbb{R}^{2}$ whose boundary has a corner at a point $O$, which without loss of generality will be considered to be at the origin $(O=(0,0))$. Further, we will consider $\partial \Omega$ to be at least piecewise smooth, that the tangent rays at the corner create an angle of size $2 \alpha$ at $O$ and these tangent rays at $O$ are $\theta= \pm \alpha$.

We will say that the corner is convex when $0 \leq \alpha<\frac{\pi}{2}$ and that the corner is non-convex 
or reentrant when $\frac{\pi}{2} \leq \alpha<\pi$. For cases when $\alpha=0$ or $\alpha=\pi$, the region is said to have a cusp. Lastly, the case of a smooth boundary with a discontinuous contact angle at point $O$ is included with $\alpha=\frac{\pi}{2}$.

Now the positive gravity case $(\kappa>0)$ with constant contact angle $\gamma$ and straight boundary segments $\partial^{ \pm} \Omega^{*}$ (refer to figure 1.2) has already been well researched. For a convex corner, Paul Concus and Robert Finn have shown that if $\left|\frac{\pi}{2}-\gamma\right|>\alpha$ then $f$ is unbounded in $\Omega^{*}$ and if $\left|\frac{\pi}{2}-\gamma\right| \leq \alpha$ then $f$ is bounded in $\Omega^{*}$. They also have shown that any solution $f$ will become unbounded at $O$ on the order of $\frac{1}{r}$. Erich Miersemann improved this estimate by showing that

$$
\left|f(r \cos (\theta), r \sin (\theta))-\frac{\cos (\theta)-\sqrt{k^{2}-\sin ^{2}(\theta)}}{k \kappa r}\right|<C r^{\epsilon}
$$

near $O$, where $k=\frac{\sin (\alpha)}{\cos (\gamma)}$. Miersemann later obtained an expansion in powers of $r$ that is asymptotic to any prescribed finite order and whose coefficients and domain are independent of the particular solution considered. Using a similar method as Miersemann's, Concus and Finn obtained an expansion in the case of a discontinuous, locally constant contact angle with the result that for any nonnegative integer $m$ there exist positive constants $r_{0}, A$, and $m+1$ bounded analytic functions $h_{4 j-1}(\theta)$ such that:

$$
\left|f(r \cos (\theta), r \sin (\theta))-\sum_{j=0}^{m} h_{4 j-1}(\theta) r^{4 j-1}\right|<A r^{4 m+3}
$$

Moreover, the constants $r_{0}, A$, and the functions $h_{4 j-1}$ are independent of the particular solution $f$ being considered. [34]

\subsection{The Concus-Finn Conjecture}

An extremely interesting and important question about nonparametric capillary surfaces is that of determining necessary and sufficient conditions for the continuity at a corner $P$ of a solution $f$ of (1.1)-(1.2). As a solution of a boundary value problem for a quasi-linear elliptic equation with positive genre $(g=2$, and thus very irregular), this possibility of obtaining 


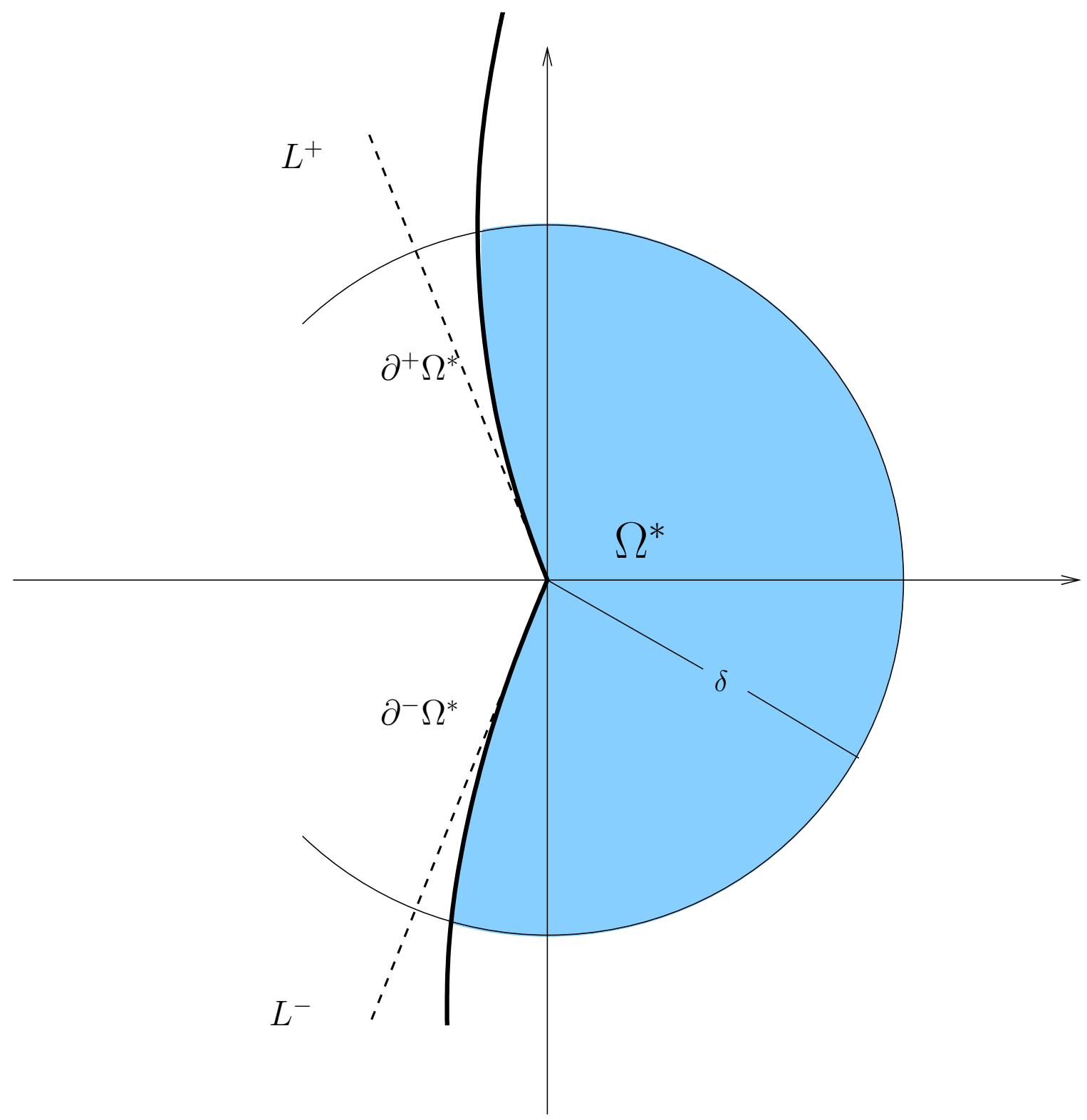

Figure 1.2: The local domain (shaded) for the reentrant corner case. $\frac{\pi}{2} \leq \alpha<\pi$ 
such conditions should have been highly unlikely; solutions of equations like (1.1) can behave near a point of the boundary of the domain in radically different ways than can solutions of equations such as Laplace's equation or a Poisson equation, as illustrated by Lancaster and Jin [25] and Serrin [49].

As stated in the previous section, we assume $\Omega$ is a domain in $\mathbb{R}^{2}$ whose boundary has a corner of size $2 \alpha$ at a point which, we take to be the origin $O=(0,0)$; we further assume that the domain is oriented such that the rays $\theta=+\alpha$ and $\theta=-\alpha$ are tangent to $\partial \Omega$ at $O$ and the directions $\theta \in(-\alpha,+\alpha)$ are the interior directions to $\Omega$ from $O$, where $(r, \theta)$ denote polar coordinates about $O$. We parameterize $\partial \Omega$ near $O$ by $(x(s), y(s))$ such that $(x(0), y(0))=(0,0), y(s)>0$ for $s>0$ and $y(s)<0$ for $s<0$. We also suppose that the contact angle $\gamma=\gamma(x, y)$ may depend on $(x, y) \in \partial \Omega$ and assume the limits

$$
\gamma_{1}=\lim _{s \downarrow 0} \gamma(x(s), y(s)) \quad \text { and } \quad \gamma_{2}=\lim _{s \uparrow 0} \gamma(x(s), y(s))
$$

both exist.

Attempts to study of capillary surfaces in wedge domains can be problematic, as illustrated by Keller (1991) and Vreeburg (1990). In 1991 Concus and Finn found that Vreeburg was incorrect; Vreeburg's argument is invalid since it does not distinguish between mean curvature and Gauss curvature and ignores the findings of Tam in 1986. We also observe that Vreeburg fails to consider the possibility of a jump discontinuity in the trace of the capillary surface on the boundary cylinder (see [37]). In 1996 Concus and Finn noted that Keller's 1991 paper was incorrect; Keller, King and Merchant argued that the only ruled minimal surfaces in $\mathbb{R}^{3}$ were planes, ignoring helicoids, and seemed to be confused about the notion of principal curvature. As with Vreeburg, they failed to consider the possibility of bounded, discontinuous (at the corner) capillary surfaces when $\gamma_{1} \neq \gamma_{2}$. Concus and Finn did consider this important possibility; as part of their investigation of these capillary surfaces, they discussed the question of continuity at a corner and in 1992 formulated the following conjecture for convex corners. [36] 


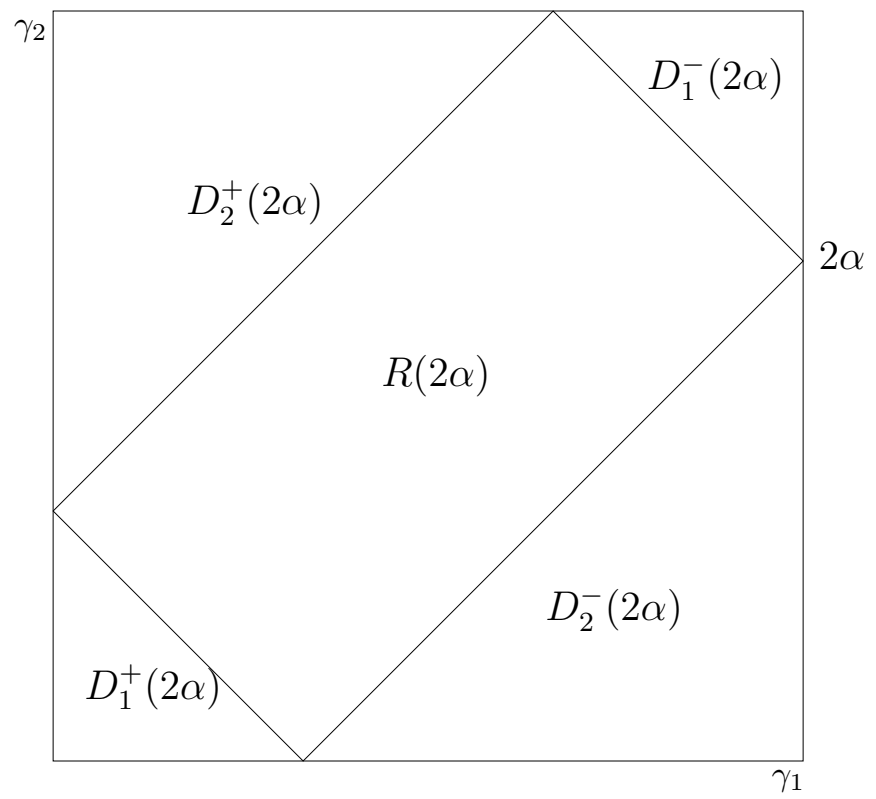

Figure 1.3: The Concus-Finn rectangle

Concus-Finn Conjecture: (e.g. [50]) Suppose $\alpha \in\left(0, \frac{\pi}{2}\right), \gamma_{1}, \gamma_{2} \in(0, \pi)$ and $\left|\gamma_{1}-\gamma_{2}\right|>$ $\pi-2 \alpha$. Then a solution of (1.1)-(1.2) must be discontinuous at $O$.

Refering to figure 1.3, for general $\gamma_{1}, \gamma_{2}$ and $\kappa=0$, if the data falls in the $D_{1}$ regions then there is no solution; for data in the $D_{2}$ solutions exist, but they have discontinuous unit normals at $O$. For data in $R$ and $2 \alpha<\pi$, all solutions are continuous. For $\gamma_{1}=\gamma_{2}=\gamma$, if $\alpha<\frac{\pi}{2}$ and $\alpha+\gamma \geq \frac{\pi}{2}$ than all solutions are differentiable up to $O$. Lieberman (1988) and Miersemann (1989) showed that such solutions are Holder differentiable at $O$. Further, Huff and McCuan (2006) gave examples to show that solutions need not be twice differentiable at $O$. When $\kappa>0$ and $\gamma_{1}=\gamma_{2}=\gamma$, for data in $D_{1}$ all solutions become unbounded at $O$ like $\frac{1}{r}$. (We note that Lancaster has proved this conjecture, which is in a paper currently submitted to the Pacific Journal of Math ([35]).) What the Concus-Finn conjecture implies is that for all data in $D_{2}$, all solutions are discontinuous. 


\subsection{Radial Limits, Central Fans and Continuity at Corners}

Lancaster and Siegel in [37] proved the existence of radial limits of a bounded solution $f$ to (1.1) that satisfies (1.2) on the sufficiently smooth portions of $\partial \Omega$ where $\gamma$ is bounded away from 0 and $\pi$, and for a convex corner an additional condition is satisfied that couples $\gamma^{+}$and $\gamma^{-}$. We denote the radial limits of $f$ by

$$
R f(\theta)=\lim _{r \rightarrow 0+} f(r \cos \theta, r \sin \theta)
$$

and

$$
R f( \pm \alpha)=\lim _{\mathbf{x} \rightarrow O, \mathbf{x} \in \partial^{ \pm} \Omega^{*}} f(\mathbf{x}), \mathbf{x}=(x, y)
$$

for $-\alpha<\theta<\alpha$, which are the limits of the boundary values of $f$ on the two sides of the corner.

We now provide, without proof (see [37] for these details), the necessary theorems that establish these radial limits exist.

Theorem 1. ([37]) Let $f$ be a bounded solution to (1.1)that satisfies (1.2)on $\partial^{ \pm} \Omega^{*} \backslash O$, which is discontinuous at $O$, with $0<\gamma_{0} \leq \gamma^{ \pm}(s) \leq \gamma_{1}<\pi$. If $\alpha \geq \pi / 2$ then $R f(\theta)$ exists for all $\theta \in[-\alpha, \alpha]$. If $\alpha<\pi / 2$ and there exist constants $\underline{\gamma}^{ \pm}, \bar{\gamma}^{ \pm}, 0<\underline{\gamma}^{ \pm} \leq \pi / 2, \pi / 2 \leq \bar{\gamma}^{ \pm}<\pi$, that satisfy

$$
\underline{\gamma}^{+}+\underline{\gamma}^{-}>\pi-2 \alpha \text { and } \bar{\gamma}^{+}+\bar{\gamma}^{-}<2 \alpha+\pi
$$

so that $\underline{\gamma}^{ \pm} \leq \gamma^{ \pm}(s) \leq \bar{\gamma}^{ \pm}$for all $s, 0<s<s_{0}$, for some $s_{0}$, then we again have $R f(\theta)$ existing for all $\theta \in[-\alpha, \alpha]$. Furthermore, in either case, $R f(\theta)$ is a continuous function on $[-\alpha, \alpha]$ who's behavior can be characterized in one of the following ways:

(i) There exist $\alpha_{1}$ and $\alpha_{2}$ such that $-\alpha \leq \alpha_{1}<\alpha_{2} \leq \alpha$, and $R f$ is constant on $\left[-\alpha, \alpha_{1}\right]$ and $\left[\alpha_{2}, \alpha\right]$, and strictly increasing or strictly decreasing on $\left[\alpha_{1}, \alpha_{2}\right]$. Label these case(I) and case(D), respectively. 


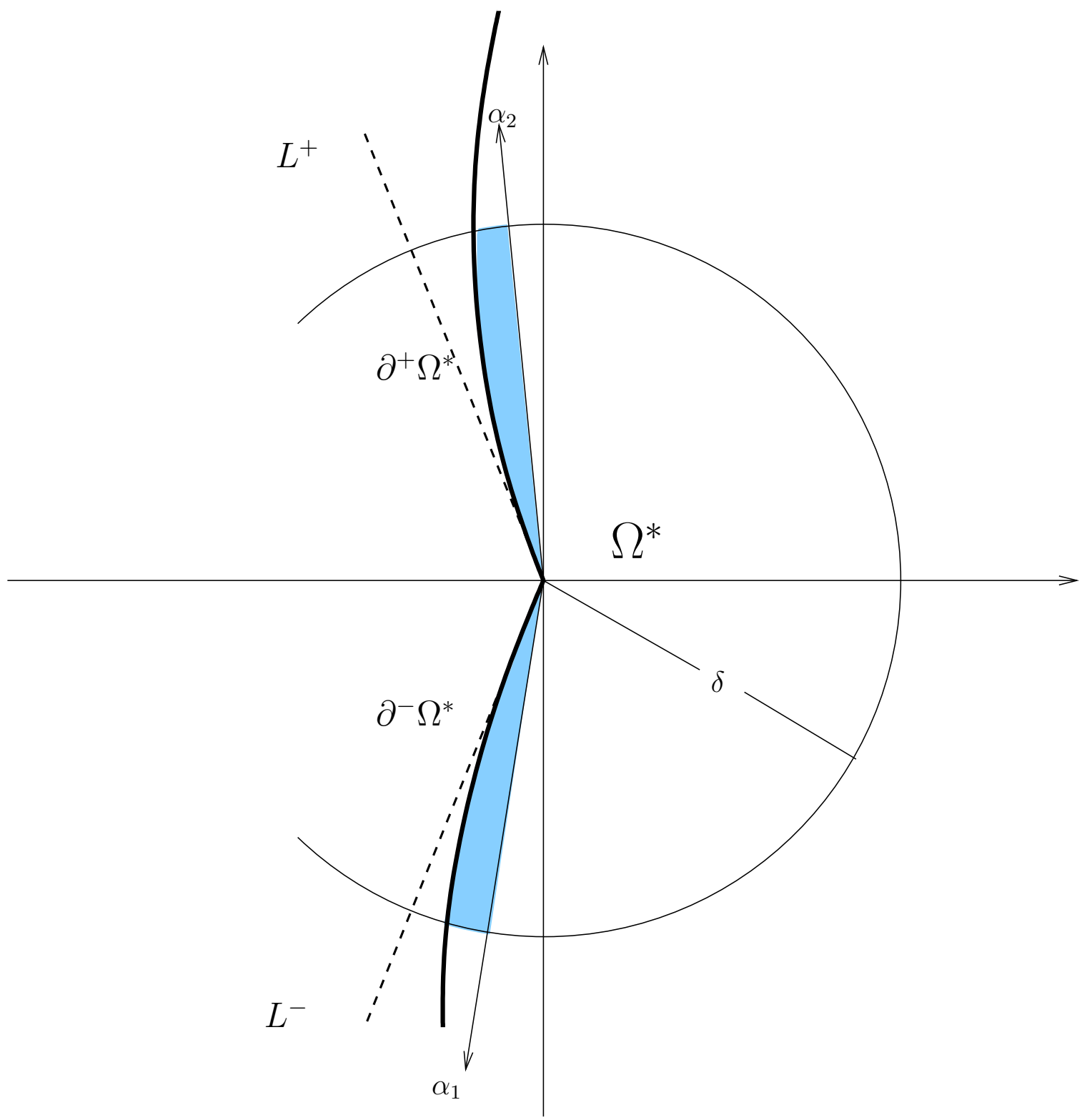

Figure 1.4: Radial Limits 
(ii) There exist $\alpha_{1}, \alpha_{L}, \alpha_{R}, \alpha_{2}$ so that $-\alpha \leq \alpha_{1}<\alpha_{L}<\alpha_{R}<\alpha_{2} \leq \alpha, \alpha_{R}=\alpha_{L}+\pi$, and $R f$ is constant on $\left[-\alpha, \alpha_{1}\right],\left[\alpha_{L}, \alpha_{R}\right]$, and $\left[\alpha_{2}, \alpha\right]$ and either increasing on $\left[\alpha_{1}, \alpha_{L}\right]$ and decreasing on $\left[\alpha_{R}, \alpha_{2}\right]$ or decreasing on $\left[\alpha_{1}, \alpha_{L}\right]$ and increasing on $\left[\alpha_{R}, \alpha_{2}\right]$. Label these case (ID) and case (DI), respectively.

When $\kappa \geq 0, f$ is automatically bounded.

Proposition 1. ([37]) Let $f$ be a solution to (1.1) satisfying (1.2) on $\partial^{ \pm} \Omega^{*} \backslash O$, with $\gamma$ satisfying the restrictions in Theorem 1 and $\kappa \geq 0$. Then $f$ is bounded in a neighborhood of O.

If as we approach $O$ the contact angle has limiting values on $\partial^{ \pm} \Omega^{*}$, we have:

Theorem 2. ([37]) In addition to the hypotheses of Theorem 1 assume that $\lim _{s \rightarrow 0+} \gamma^{ \pm}(s)=$ $\gamma_{0}^{ \pm}$both exist. Then there exist fans of constant radial limits adjacent to each tangent direction at $O: \alpha_{1}+\alpha \geq \gamma_{0}^{-}$or $\pi-\gamma_{0}^{-}$and $\alpha-\alpha_{2} \geq \gamma_{0}^{+}$or $\pi-\gamma_{0}^{+}$. The lower bound on the size of which the fan holds is described in terms of the cases labeled in Theorem 1:

$$
\begin{aligned}
& \alpha_{1}+\alpha \geq \gamma_{0}^{-} \text {for }(D) \text { and }(D I) \\
& \alpha_{1}+\alpha \geq \pi-\gamma_{0}^{-} \text {for }(I) \text { and }(I D) \\
& \alpha-\alpha_{2} \geq \gamma_{0}^{+} \text {for }(I) \text { and }(D I) \\
& \alpha-\alpha_{2} \geq \pi-\gamma_{0}^{+} \text {for }(D) \text { and (ID) }
\end{aligned}
$$

One of the results obtained from [37] (and restated in [36]), specifically from Corollaries 2 and 3 , is the non-convex (reentrant) analog to the Concus-Finn Rectangle (figure 1.3):

- For $\gamma_{1}, \gamma_{2} \in R(2 \alpha), f$ is continuous up to $O$, or be in case (D) or (I).

- For $\gamma_{1}, \gamma_{2} \in D_{1}^{+}(2 \alpha), f$ cannot be in case (ID.)

- For $\gamma_{1}, \gamma_{2} \in D_{1}^{-}(2 \alpha), f$ cannot be in case (DI.)

- For $\gamma_{1}, \gamma_{2} \in D_{2}^{+}(2 \alpha), f$ can be continuous up to $O$ or be in case (D).

- For $\gamma_{1}, \gamma_{2} \in D_{2}^{-}(2 \alpha), f$ can be continuous up to $O$ or be in case (I). 
In short, there are no continuous solutions in the $D_{1}^{ \pm}$or $D_{2}^{ \pm}$regions. This result was proved by Shi in [50].

As noted earlier, in a response to [37], Shi and Finn [51] found that the central fan of radial limits was very much dependant upon symmetry phenomena. To quote the abstract of [51]: "In establishing conditions for continuity of the height of a capillary surface $f(x, y)$ at a re-entrant corner point of the domain of definition, Lancaster and Siegel introduced a hypothesis of symmetry, which does not appear in corresponding conditions for a protruding corner. We show here that the hypothesis cannot be discarded. Starting with a symmetric configuration for which the surface height is continuous at the corner point in accordance with the hypotheses of those authors, we show that the height can be made discontinuous by an asymmetric domain perturbation that is in an asymptotic sense arbitrarily small, and for which all hypotheses other than that of symmetry remain in force."

\subsubsection{Convex Corners}

In Corollary 4 of [37], Lancaster and Siegel proved that a solution $f$ of (1.1)-(1.2) must be continuous at $O$ if $\alpha \leq \frac{\pi}{2},\left|\gamma_{1}-\gamma_{2}\right| \leq \pi-2 \alpha$ and $\left|\gamma_{1}+\gamma_{2}-\pi\right|<2 \alpha$. The proof of Corollary 4 follows from lower bounds on the sizes of "fans" of constant radial limits obtained in Theorem 2 of [37]. These bounds implied the "fans" touch or overlap and hence $R f$ is a constant function on $[-\alpha, \alpha]$, where $R f( \pm \alpha)$ are defined as the one-sided limits at $O$ of the trace of $f$ and these limits are proven to exist as part of the proof of Theorem 1 ; if $f$ is discontinuous at $O$, Theorem 1 implies $R f$ is not constant. When $\left|\gamma_{1}+\gamma_{2}-\pi\right|>2 \alpha$, we know that no solution of (1.1)-(1.2) can remain bounded near $O$ (see [5], [6]) and when $\left|\gamma_{1}+\gamma_{2}-\pi\right|=2 \alpha$, a variation of Tam's argument from his 1986 work should establish the continuity of a solution at $O$. Since the Concus-Finn conjecture was proved, we have that $f$ is discontinuous at the corner if $\left|\gamma_{1}-\gamma_{2}\right|>\pi-2 \alpha$. Thus, assuming the successful application of Tam's arguments, the continuity or discontinuity of a solution of (1.1)-(1.2) at a convex corner is completely determined by "local" geometric information (i.e. $\gamma_{1}, \gamma_{2}$ and $\alpha$ ); when 
$\alpha \leq \frac{\pi}{2}, f$ is continuous at $O$ if and only if $\left|\gamma_{1}+\gamma_{2}-\pi\right| \leq 2 \alpha$ and $\left|\gamma_{1}-\gamma_{2}\right| \leq \pi-2 \alpha$. [36]

\subsubsection{Nonconvex Corners}

Recall that conclusion of Theorem 1 of [37] is that the radial limits of $f$ behave in one of the following ways:

(i) There exist $\alpha_{1}$ and $\alpha_{2}$ so that $-\alpha \leq \alpha_{1}<\alpha_{2} \leq \alpha$ and $R f$ is constant on $\left[-\alpha, \alpha_{1}\right]$ and $\left[\alpha_{2}, \alpha\right]$ and strictly increasing or strictly decreasing on $\left[\alpha_{1}, \alpha_{2}\right]$.

(ii) There exist $\alpha_{1}, \alpha_{L}, \alpha_{R}, \alpha_{2}$ so that $-\alpha \leq \alpha_{1}<\alpha_{L}<\alpha_{R}<\alpha_{2} \leq \alpha, \alpha_{R}=\alpha_{L}+\pi$, and $R h$ is constant on $\left[-\alpha, \alpha_{1}\right],\left[\alpha_{L}, \alpha_{R}\right]$, and $\left[\alpha_{2}, \alpha\right]$ and either strictly increasing on $\left[\alpha_{1}, \alpha_{L}\right]$ and strictly decreasing on $\left[\alpha_{R}, \alpha_{2}\right]$ or strictly decreasing on $\left[\alpha_{1}, \alpha_{L}\right]$ and strictly increasing on $\left[\alpha_{R}, \alpha_{2}\right]$.

Notice that Theorem 1 (and its proof) shows that the size of the "central fan" $\left[\alpha_{L}, \alpha_{R}\right]$ of directions for which $R f$ is constant is exactly $\pi$. For the $\kappa=\lambda=0$ in (2.1), the fact that the size of the central fan is exactly $\pi$ was obtained in Theorem 2 of [31]. Further, notice that the conclusion of Theorem 2 (above) is that there are lower bounds on the sizes of the "side fans" $\left[-\alpha, \alpha_{1}\right]$ and $\left[\alpha_{2}, \alpha\right]$ depending on which of the cases (I), (D), (ID) or (DI) holds, and these bounds are:

$$
\begin{aligned}
& \alpha_{1}+\alpha \geq \gamma_{2} \text { for (D) and (DI); } \\
& \alpha_{1}+\alpha \geq \pi-\gamma_{2} \text { for (I) and (ID); } \\
& \alpha-\alpha_{2} \geq \gamma_{1} \text { for (I) and (DI); } \\
& \alpha-\alpha_{2} \geq \pi-\gamma_{1} \text { for (D) and (ID). }
\end{aligned}
$$

The existence of fans of radial limits, particularly central fans, is where our interest lie. Example 2 of [37] is a nonparametric capillary surface where a central fan can be seen to exist. In [50], Shi assumes the Concus-Finn Conjecture holds and proves that for $\kappa>0$, $\alpha>\frac{\pi}{2}$ and either $\left|\gamma_{1}-\gamma_{2}\right|>2 \alpha-\pi$ or $\left|\gamma_{1}+\gamma_{2}-\pi\right|>2 \pi-2 \alpha$, then a solution $f$ of (1.1)-(1.2) must be discontinuous at $O$. With the proof of the Concus-Finn Conjecture, this means that 
the continuity question at a non-convex corner reduces to the situation where the size $2 \alpha$ of the opening angle and the limiting contact angles $\gamma_{1}$ and $\gamma_{2}$ are related by

$$
\left|\gamma_{1}-\gamma_{2}\right| \leq 2 \alpha-\pi \quad \text { and } \quad\left|\gamma_{1}+\gamma_{2}-\pi\right| \leq 2 \pi-2 \alpha
$$

When $\left|\gamma_{1}+\gamma_{2}-\pi\right| \leq 2 \pi-2 \alpha$, Lancaster and Athanassenas ( [36]) define the central fan question to be the issue of finding necessary and sufficient conditions for excluding Theorem 1 (i) of [37] (i.e. case (I) and case (D), the monotonic cases). They demonstrate that the only possibilities are that $f$ is discontinuous at the corner $O$ and Theorem 1(i) holds at $O$ or that $f$ is continuous at $O$. To see this, suppose $f$ is discontinuous at $O$ and either case (ID) or (DI) holds. If (ID) holds, then $\alpha-\alpha_{2} \geq \pi-\gamma_{1}, \alpha_{1}-(-\alpha) \geq \pi-\gamma_{2}$ and (using the fact that central fans are of size $\pi$ ) the sum of the sizes of the three fans is at least $\pi-\gamma_{1}+\pi+\pi-\gamma_{2}=3 \pi-\gamma_{1}-\gamma_{2}$. Now if (DI) holds, then $\alpha-\alpha_{2} \geq \gamma_{1}, \alpha_{1}-(-\alpha) \geq \gamma_{2}$ and the sum of the sizes of the three fans is at least $\gamma_{1}+\pi+\gamma_{2}$. Now $\left|\gamma_{1}+\gamma_{2}-\pi\right| \leq 2 \pi-2 \alpha$ implies $2 \alpha \leq \pi+\gamma_{1}+\gamma_{2}$ and $2 \alpha \leq 3 \pi-\gamma_{1}-\gamma_{2}$ and these imply that the sum of the sizes of the fans is at least $2 \alpha$; since $R f$ is constant on each fan, $R f$ must be constant and therefore the assumption that $f$ is discontinuous at $O$ is must be false. Thus, we see that a necessary and sufficient condition for the continuity of $f$ at $O$ is that $\left|\gamma_{1}+\gamma_{2}-\pi\right| \leq 2 \pi-2 \alpha$ and the radial limits of $f$ are be monotonic (or, in other words, Theorem 1(i), of [37] hold).

Consider the symmetry condition [36] that says that a capillary problem (1.1)-(1.2) satisfies an "(even) symmetry condition at a point $P \in \partial \Omega$ " if there is a rigid motion $L: \mathbb{R}^{2} \rightarrow \mathbb{R}^{2}$ such that $L(P)=O, L(\Omega)$ is symmetric with respect to the $x$-axis and $\gamma \circ L^{-1}(x,-y)=\gamma \circ L^{-1}(x, y)$ for all $(x, y) \in L(\partial \Omega)$.

Corollary 2 of [37] is the first result which provides sufficient conditions for the existence of a central fan; from this follows: Suppose $\kappa \geq 0, \Omega$ has a non-convex corner at $P \in \partial \Omega,(1.1)$ (1.2) satisfies the symmetry condition at $O$ and $f$ satisfies (1.1)-(1.2). Then $f^{*}=f \circ L^{-1}$ is even in $y$ and $R f^{*}(\theta ; O)$ is constant for $\theta \in\left[-\frac{\pi}{2}, \frac{\pi}{2}\right]$. 
As stated previously, in [51], Shi and Finn examined Example 2 of [37] and modified its construction to obtain a $\kappa>0$ capillary surface in an asymmetric domain; their solution satisfied the angle requirements of Corollary 2 of [37] (i.e. (1.7) with $\gamma_{1}=\gamma_{2}$ ) but was discontinuous at $O$. The Shi-Finn construction made, in a sense explained in [51], an arbitrarily small perturbation in the domain which left a neighborhood of $O$ unaltered. Thus a perturbation in the domain well away from the corner causes a discontinuity in $f$ at $O$, and therefore the symmetry condition is required for continuity.

Wishing to observe these fans of radial limits, we will review the procedures necessary to numerically construct surfaces of the type used in [36] by computing conformal maps and calculating a few complex integrals. These procedures will be discussed in the next chapter, and are the basis for Francis Nguyen's computations in [47].

\subsection{Constructing a Capillary Graph}

As in [36] our domain construction begins by assuming that domain $\Omega$ is symmetric about the coordinate axes, and star shaped as in figure (1.5). In each quadrant, $\partial \Omega$ has a non-convex corner, and their interest is in the existence of the fans of radial limits at these corners. They remind the reader that they must keep in mind that the symmetry condition will not hold at these corners when $\gamma_{1} \neq \gamma_{2}$ (for example, $f$ will not be symmetric with respect to the line through point $P$ that bisects angle $A P B$ in figure (1.5) when $\gamma_{1} \neq \gamma_{2}$ ). The reader's attention is restricted to $\Omega_{0}$, which is defined as the quarter of the domain in the second quadrant, and require the Gauss map of the surface $z=f$ over $\Omega_{0}$ to be injective. While this requirement that the Gauss map be injective is critical for their construction using the Weierstrass $(f, g)$-representation; minimal surfaces with injective Gauss maps over nonconvex domains are, in some sense, rare and this requirement will "single out" (dependant upon $\gamma$ ) the particular domain $\Omega$ in which we will work. [36]

Specifically, to create the domain, they let $\delta \in\left(0, \frac{\pi}{4}\right)$ and, for $B=\left(x_{0}, 0\right)$ with $x_{0} \in$ $(-\cot (\delta),-\tan (\delta))$, consider the domain $\Omega(B) \subset \mathbb{R}^{2}$, as illustrated in Figure (1.5), whose 


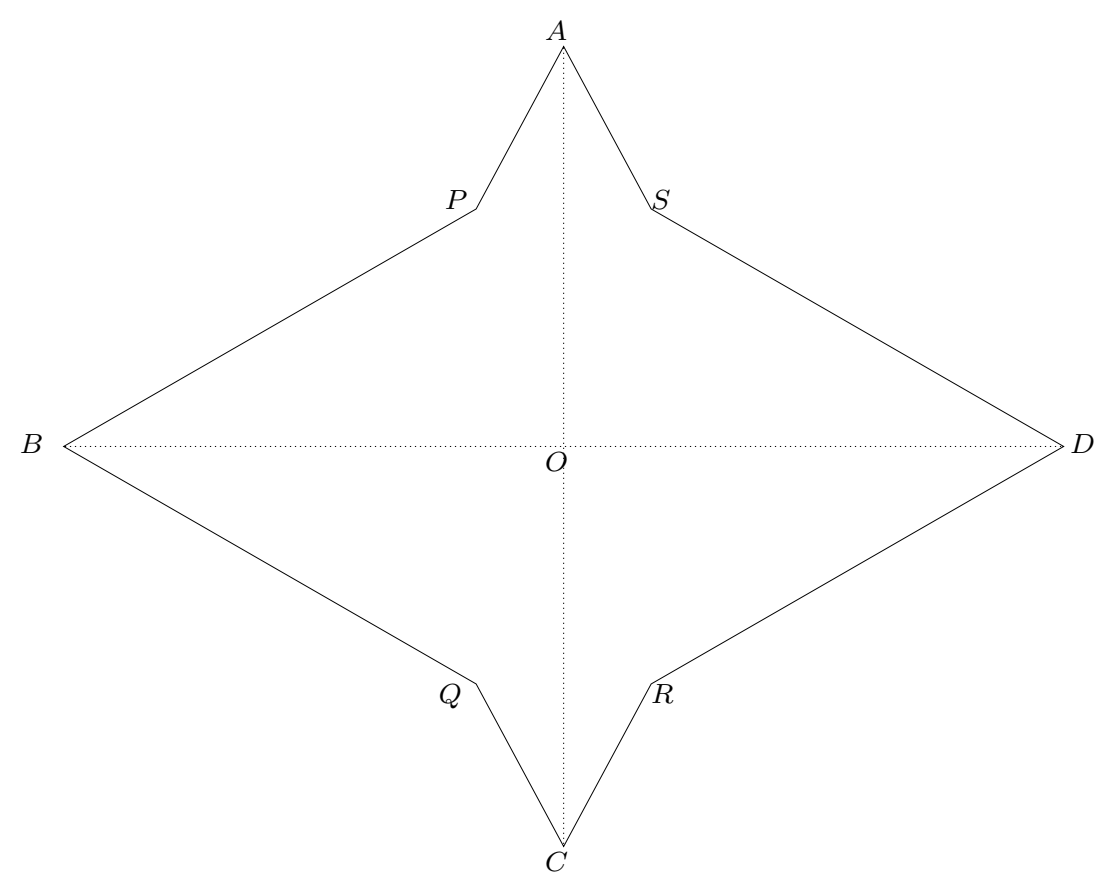

Figure 1.5: $\Omega$

boundary is a polygon with eight sides which is symmetric with respect to the horizontal and vertical axes and whose two sides in the second quadrant have vertices at $(0,1), P$ and $B$, where $P=P\left(x_{0}\right)$ is the point of intersection of the lines $y=\cot (\delta) x+1$ and $y=\tan (\delta)\left(x-x_{0}\right)$. In this figure, Lancaster and Athanassenas have labeled the vertices of $\partial \Omega$, starting at $(0,1)$ and moving in a counterclockwise direction, as $A, P, B, Q, C, R, D$ and $S$ respectively and label the origin as $O$. Notice that the convex angles $O A P$ and $O B P$ have measure $\delta$ and the non-convex angle $A P B$ has measure $\frac{3}{2} \pi-2 \delta$. Let $\Omega_{0}(B)$ be the (open) region whose boundary is the non-convex quadrilateral $A P B O$.

Consider $\Omega=\Omega(B)$ as the cross section of a vertical cylinder $\Lambda$. Here, the authors must specify the contact angle boundary condition on $\Lambda$. Let $\gamma_{1}, \gamma_{2} \in\left(0, \frac{\pi}{2}\right)$ satisfy the condition

$$
\tan (\delta)<\frac{\cos \left(\gamma_{2}\right)}{\cos \left(\gamma_{1}\right)}<\cot (\delta)
$$


and define the function $\gamma: \partial \Omega \rightarrow \mathbb{R}$ by

$$
\gamma(x, y)=\left\{\begin{array}{cl}
\gamma_{1} & \text { if }(x, y) \in A P \cup C R \\
\gamma_{2} & \text { if }(x, y) \in P B \cup R D \\
\pi-\gamma_{2} & \text { if }(x, y) \in B Q \cup D S \\
\pi-\gamma_{1} & \text { if }(x, y) \in Q C \cup S A .
\end{array}\right.
$$

Now, consider the capillary problem (1.1)-(1.2) in cylinder $\Lambda$ with contact angle boundary data $\gamma$, gravitational constant $\kappa \geq 0$ and Lagrange multiplier $\lambda$. In [36], they are interested in the behavior of the solution in zero gravity, and so shall we be. In this case, the combination of $\kappa=0$, the divergence theorem, and equations (1.1) and (1.2) implies

$$
\lambda|\Omega|=\int_{\Omega} \operatorname{div}(T f) d A=\int_{\partial \Omega} \cos (\gamma) d s .
$$

Since $\cos (\gamma(x, y))$ is an odd function of $x$ and of $y$, we see that $\lambda=0$. This means the solution surface $f$ will be minimal, as required.

For their study, Lancaster and Athanassenas consider pairs $\left(\gamma_{1}, \gamma_{2}\right)$ of contact angles which satisfy the additional restrictions of $\gamma_{1} \geq \delta$ and $\gamma_{2} \geq \delta$ for $\delta \in\left(0, \frac{\pi}{4}\right)$. The authors want the stereographic projection $E$ of the image of the Gauss map of their solution over $\overline{\Omega_{0}(B)}$ for an appropriate $B$, and this will be the region that will need to be conformally mapped to the disc. To determine $E$, let $B_{1}=\{w \in \mathbb{C}:|w|<1\}, Q_{1}=\left\{w \in B_{1}: \operatorname{Re}(w)<0, \operatorname{Im}(w)>0\right\}$ and set

$$
E_{0}=\left\{w \in Q_{1}:\left|w-w_{1}\right|>\tan \left(\gamma_{1}\right),\left|w-w_{2}\right|>\tan \left(\gamma_{2}\right)\right\}
$$

where $w_{1}=u_{1}+i v_{1}=-\cos (\delta) \sec \left(\gamma_{1}\right)+i \sin (\delta) \sec \left(\gamma_{1}\right)$ and $w_{2}=u_{2}+i v_{2}=-\sin (\delta) \sec \left(\gamma_{2}\right)+$ $i \cos (\delta) \sec \left(\gamma_{2}\right)$. Now, the constant contact angle condition on the vertical plane $\overline{A P} \times \mathbb{R}$ corresponds to requiring the Gauss map to lie on the circle on $S^{2}$ which is the intersection of 
$S^{2}$ and a cone with opening angle $\gamma_{1}$. The stereographic projection of this circle is the circle

$$
C_{1}=\left\{w:\left|w-w_{1}\right|=\tan \left(\gamma_{1}\right)\right\}
$$

centered at $w_{1}$. In the same way, the authors point out that the constant contact angle condition on the vertical plane $\overline{P B} \times \mathbb{R}$ corresponds to requiring the Gauss map to lie on the circle on $S^{2}$ which is the intersection of $S^{2}$ and a cone with opening angle $\gamma_{2}$. The stereographic projection of this second circle is

$$
C_{2}=\left\{w:\left|w-w_{2}\right|=\tan \left(\gamma_{2}\right)\right\} .
$$

Then $E=\overline{E_{0}}$.

Now let's take a look at set $E$, which is a simply connected subset of the closed unit disc. The boundary of $E$ consists of portions of the circles $C_{1}$ and $C_{2}$ which are orthogonal to the unit circle $\partial B_{1}$, the positive imaginary axis, the negative real axis and (possibly) the unit quarter-circle $\left\{w \in \partial B_{1}: \operatorname{Re}(w) \leq 0, \operatorname{Im}(w) \geq 0\right\}$. If the circles $C_{1}$ and $C_{2}$ intersect in $|w| \leq 1$, then let $w_{0}=u_{0}+i v_{0}$ be this point of intersection; (1.8) implies $w_{0} \in Q_{1}$ and by reflecting about $\partial B_{1}$, we see that

$$
\left(v_{2}-v_{1}\right) v_{0}=\left(u_{1}-u_{2}\right) u_{0} .
$$

Note that $C_{1}$ lies entirely in the open set $\operatorname{Re}(w)<0$ for $\gamma_{1}<\frac{\pi}{2}-\delta$ and $C_{2}$ lies entirely in the open set $\operatorname{Im}(w)>0$ for $\gamma_{2}<\frac{\pi}{2}-\delta$.

Let sets $\sigma_{j}, j=1,2, \ldots 5$ be defined as

$$
\begin{gathered}
\sigma_{1}=\partial E \cap C_{1}, \quad \sigma_{2}=\partial E \cap C_{2}, \quad \sigma_{3}=\{w \in \partial E: \operatorname{Im}(w)=0\} \\
\sigma_{4}=\{w \in \partial E \quad: \operatorname{Re}(w)=0\} \quad \& \quad \sigma_{5}=\{w \in \partial E:|w|=1\} .
\end{gathered}
$$




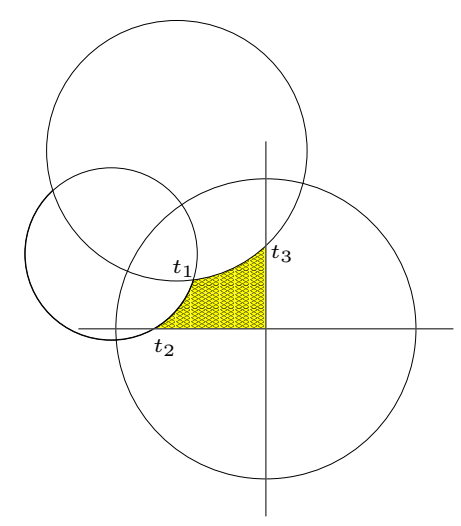

Figure 1.6: $\gamma_{1} \geq \delta, \gamma_{2} \geq \delta, \gamma_{1}+\gamma_{2} \geq \frac{\pi}{2}-2 \delta$

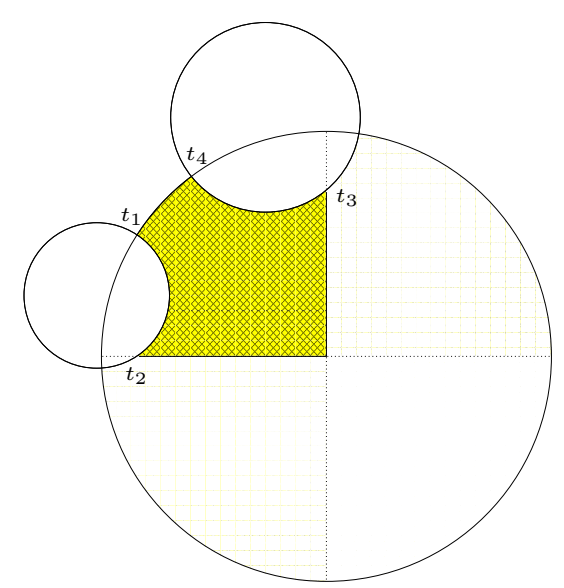

Figure 1.7: $\gamma_{1} \geq \delta, \gamma_{2} \geq \delta, \gamma_{1}+\gamma_{2}<\frac{\pi}{2}-2 \delta$

Denote vertex $t_{0}=0$. Denote the remaining vertices of the polygon formed by $\partial E$ and the $\sigma_{j}$ as $t_{1}, \ldots t_{k}$ for $3 \leq k \leq 4$. These $t_{i}$ have the following properties:

(i) $t_{1}, t_{2} \in \sigma_{1}$ and $\operatorname{Im}\left(t_{2}\right)=0$; i.e. $t_{2}$ is the intersection of $C_{1}$ with the negative real axis and $t_{1}$ is the intersection of $C_{1}$ with either $C_{2}$ (see (iii)) or $\partial B_{1}$ (see (iv)).

(ii) $t_{3} \in \sigma_{2}$ and $\operatorname{Re}\left(t_{3}\right)=0$; i.e. $t_{3}$ is the intersection of $C_{2}$ with the positive imaginary axis.

(iii) $\gamma_{1}+\gamma_{2} \geq \frac{\pi}{2}-2 \delta$ implies $k=3$ and $t_{1} \in \sigma_{2}$;

(iv) $\gamma_{1}+\gamma_{2}<\frac{\pi}{2}-2 \delta$ implies $k=4, t_{4} \in \sigma_{2}$ and $t_{1}, t_{4} \in \partial B_{1}$.

When condition (iii) holds, figure 1.6 illustrates the shape of $E$,. While figure 1.7 illustrates the shape of $E$ when condition (iv) holds. Note that when condition (iv) holds, $t_{1}=e^{\left(\pi-\delta-\gamma_{1}\right) i}$ and $t_{4}=e^{\left(\frac{\pi}{2}+\delta+\gamma_{2}\right) i}$. 
For the Weierstrass representation, let us define $g: E \rightarrow E$ by $g(w)=w$. It is necessary to find $f \in C^{0}\left(E \backslash\left\{t_{1}, \ldots, t_{k}\right\}\right)$ with, at worst, integrable singularities at $t_{1}, \ldots, t_{k}$ which is analytic in $E_{0}$ and define $X \in C^{0}\left(E: \mathbf{R}^{3}\right) \cap C^{2}\left(E_{0}: \mathbf{R}^{3}\right)$ with

$$
X(u+i v)=(x(u, v), y(u, v), z(u, v))
$$

and $K(u+i v)=(x(u, v), y(u, v))$ for $u+i v \in E$ such that $X(0)=(0,0,0)$, (a) the analytic functions $(f, g)$ form the Weierstrass representation of $X$ (Osserman [48])and require the use of fundamental forms. I detail computing fundamental forms in my Master's Thesis [42]:

(b) $K$ is a homeomorphism between $\sigma_{1}$ and $A P, \sigma_{2}$ and $P B, \sigma_{3}$ and $O A$, and $\sigma_{4}$ and $B O$ respectively (where, for example, $O A$ means the line segment between $O$ and $A$ ), and (c) $\mathrm{K}$ is constant on each component of $\sigma_{5}$.

Here we say $f$ has an integrable singularity at $t_{n}$ if and only if $|f(w)| \leq C\left|w-t_{n}\right|^{s}$ with $-1<s<0$ and $C \geq 0$ for $w$ near $t_{n}$.

At this point, the solution to a Riemann-Hilbert problem is necessary as is the rest of the outline of the numerical scheme (as computed in [47], which is shown in the next chapter. 


\section{Chapter 2}

\section{Riemann-Hilbert and the Scheme of Things}

\subsection{Solving the Riemann-Hilbert Problem}

Following the method found in [36], we begin by formulating the Riemann-Hilbert problem that must be solved by temporarily assuming the existence of a suitable function $f$. The boundary requirements (b) imply

$$
\begin{aligned}
& y(u, v)=\cot (\delta) x(u, v)+1, \quad \text { for } u+i v \in \sigma_{1} \\
& y(u, v)=\tan (\delta)\left(x(u, v)-x_{0}\right) \quad \text { for } u+i v \in \sigma_{2} \\
& x(u, v)=0 \text { and } 0 \leq y(u, v) \leq 1 \quad \text { for } u+i v \in \sigma_{3} \\
& y(u, v)=0 \text { and } \quad x_{0} \leq x(u, v) \leq 0 \quad \text { for } u+i v \in \sigma_{4},
\end{aligned}
$$

where $x_{0} \in(-\cot (\delta),-\tan (\delta))$ remains to be determined. Let $f(u+i v)=f_{1}(u, v)+i f_{2}(u, v)$, where $f_{1}$ and $f_{2}$ are real-valued. Now (a) implies

$$
\begin{aligned}
& x_{w}=f(w)\left(1-w^{2}\right) / 2 \\
& y_{w}=i f(w)\left(1+w^{2}\right) / 2 \\
& z_{w}=w f(w)
\end{aligned}
$$

for $w \in E$ (e.g. Osserman [48]). 
Since $\frac{d}{d w}=\frac{1}{2}\left(\frac{\partial}{\partial u}-i \frac{\partial}{\partial v}\right)$, the equations above yield

$$
\begin{aligned}
& x_{u}(u, v)=\operatorname{Re}\left(f(w)\left(1-w^{2}\right)\right), \\
& x_{v}(u, v)=-\operatorname{Im}\left(f(w)\left(1-w^{2}\right)\right), \\
& y_{u}(u, v)=\operatorname{Re}\left(i f(w)\left(1+w^{2}\right)\right), \\
& y_{v}(u, v)=-\operatorname{Im}\left(i f(w)\left(1+w^{2}\right)\right), \\
& z_{u}(u, v)=\operatorname{Re}(2 w f(w)) \\
& z_{v}(u, v)=-\operatorname{Im}(2 w f(w))
\end{aligned}
$$

where $w=u+i v$.

By parameterizing $\sigma_{k}$ such that $\sigma_{k}=\left\{w_{k}(t)=u_{k}(t)+i v_{k}(t): t \in I_{k}\right\}$, for $I_{k}$ a closed interval in $\mathbb{R}, k=1, \ldots, 5$, then by differentiating with respect to $t$ (and denoting $\frac{d}{d t}$ by a prime), they find

$$
\begin{aligned}
& (2) \Rightarrow y_{u} u_{1}^{\prime}+y_{v} v_{1}^{\prime}=\cot (\delta)\left(x_{u} u_{1}^{\prime}+x_{v} v_{1}^{\prime}\right), \\
& (3) \Rightarrow y_{u} u_{2}^{\prime}+y_{v} v_{2}^{\prime}=\tan (\delta)\left(x_{u} u_{2}^{\prime}+x_{v} v_{2}^{\prime}\right), \\
& (3) \Rightarrow x_{u}\left(u_{3}(t), 0\right)=0, \\
& (2.4) \Rightarrow y_{v}\left(0, v_{4}(t)\right)=0, \\
& (\mathbf{c}) \Rightarrow x_{u} u_{5}^{\prime}+x_{v} v_{5}^{\prime}=0 \text { and } y_{u} u_{5}^{\prime}+y_{v} v_{5}^{\prime}=0
\end{aligned}
$$

For $\sigma_{1}$, they have $\left(u_{1}(t)-u_{1}\right)^{2}+\left(v_{1}(t)-v_{1}\right)^{2}=r_{1}^{2}$ which implies

$$
\frac{u_{1}^{\prime}(t)}{v_{1}^{\prime}(t)}=-\frac{v_{1}(t)-v_{1}}{u_{1}(t)-u_{1}}=-\frac{\operatorname{Im}\left(w_{1}(t)-w_{1}\right)}{\operatorname{Re}\left(w_{1}(t)-w_{1}\right)}
$$

For $\sigma_{2}$, they have $\left(u_{2}(t)-u_{2}\right)^{2}+\left(v_{2}(t)-v_{2}\right)^{2}=r_{2}^{2}$ and so

$$
\frac{u_{2}^{\prime}(t)}{v_{2}^{\prime}(t)}=-\frac{v_{2}(t)-v_{2}}{u_{2}(t)-u_{2}}=-\frac{\operatorname{Im}\left(w_{2}(t)-w_{2}\right)}{\operatorname{Re}\left(w_{2}(t)-w_{2}\right)}
$$

Recall that $\cot (\delta)=-\frac{\operatorname{Re}\left(w_{1}\right)}{\operatorname{Im}\left(w_{1}\right)}=-\frac{u_{1}}{v_{1}}$ and $\tan (\delta)=-\frac{\operatorname{Re}\left(w_{2}\right)}{\operatorname{Im}\left(w_{2}\right)}=-\frac{u_{2}}{v_{2}}$. 
Applying this information, the following is obtained:

$$
\operatorname{Re}\left(\left(a_{k}(u, v)+i b_{k}(u, v)\right) f(u+i v)\right)=0,
$$

when $(u, v) \in \sigma_{k}$, for $k=1, \ldots, 5$, where

$$
\begin{gathered}
a_{1}(u, v)+i b_{1}(u, v)=i e^{i \delta}\left(w-w_{1}\right)\left(e^{-2 \delta i}-w^{2}\right) \quad \text { if } \quad w=u+i v \in \sigma_{1} ; \\
a_{2}(u, v)+i b_{2}(u, v)=e^{-i \delta}\left(w-w_{2}\right)\left(e^{2 \delta i}+w^{2}\right) \quad \text { if } \quad w=u+i v \in \sigma_{2} ; \\
a_{3}(u, v)+i b_{3}(u, v)=-1 \quad \text { if } \quad u+i v \in \sigma_{3} ; \\
a_{4}(u, v)+i b_{4}(u, v)=-1 \quad \text { if } \quad u+i v \in \sigma_{4} ;
\end{gathered}
$$

and

$$
a_{5}(u, v)+i b_{5}(u, v)=(u+i v)^{2} \quad \text { if } \quad w=u+i v \in \sigma_{5} .
$$

Now define $a, b: \partial E \rightarrow \mathbb{R}$ by $a(u+i v)=a_{k}(u, v)$ and $b(u+i v)=b_{k}(u, v)$ if $u+i v \in \sigma_{k}$, for $k \in\{1, \ldots, 5\}$ and define $G: \partial E \rightarrow \mathbb{C}$ by

$$
G(w)=a(w)+i b(w) .
$$

Note that it is possible that $\sigma_{5}=\emptyset$, in which case $a_{5}(u, v)+i b_{5}(u, v)$ has no impact on $G$.

Assuming the existence of $f$, the reader is led to (2.5). Now the authors formulate the problem of finding $X$ with $g$ equal to the identity function. Lancaster and Athanassenas wish to find a function $f \in C^{0}\left(E \backslash\left\{t_{1}, \ldots, t_{k}\right\}\right)$ which is analytic in $E_{0}$ and satisfies

$$
\operatorname{Re}(G(w) f(w))=0 \quad \text { for } \quad w \in \partial E \backslash\left\{t_{1}, \ldots, t_{k}\right\}
$$

This is a Hilbert problem with piecewise discontinuous Hölder coefficients $G$ in the notation of Monakhov [44], Chapter $1, \S 4$ (see this reference for notation). In order to use the results 
in Monakhov [44], we need to compute the index of $G$ in an appropriate function class $O(m)=O\left(t_{n_{1}}, \ldots, t_{n_{m}}\right)$ for some $m \in\{0, \ldots, k\}$. We will continue to follow Lancaster and Athanassenas in [36] as an example of this process.

Define $G_{1}: \partial E \rightarrow \mathbb{C}$ by

$$
G_{1}(w)=-\frac{\overline{G(w)}}{G(w)}
$$

And so $G_{1}(w)=-1$ for $w \in \sigma_{3} \cup \sigma_{4}, G_{1}$ is continuous at 0 and $G_{1}(w)=-\left(\frac{\bar{w}}{|w|}\right)^{4}=-\bar{w}^{4}$ for $w \in \sigma_{5}$. Set $\omega=e^{i \delta}$. Notice also that

$$
G_{1}(w)=|G(w)|^{-2}\left(\overline{\omega\left(w-w_{1}\right)\left(\bar{\omega}^{2}-w^{2}\right)}\right)^{2}, \quad \text { for } \quad w \in \sigma_{1}
$$

and

$$
G_{1}(w)=-|G(w)|^{-2} e^{2 \delta i}\left(\overline{\left(w-w_{2}\right)\left(w^{2}+\omega^{2}\right)}\right)^{2}, \quad \text { for } \quad w \in \sigma_{2} .
$$

For two corners $t_{n}$ and $t_{n+1}(n \geq 1)$ on $\partial E$, let $\left[t_{n}, t_{n+1}\right]$ denote the arc of $\partial E$ between $t_{n}$ and $t_{n+1}$ with $t_{k+1}$ denoting $t_{1}$; for example, $\left[t_{1}, t_{2}\right]=\sigma_{1}$ and $\left[t_{2}, t_{3}\right]=\sigma_{3} \cup \sigma_{4}$. For $n \in\{1, \ldots, k\}$, let

$$
\theta_{n}=\frac{1}{2 \pi}\left(\arg \left(G_{1}\left(t_{n}-0\right)\right)-\arg \left(G_{1}\left(t_{n}+0\right)\right)\right)
$$

where $\arg \left(G_{1}\left(t_{n}-0\right)\right)$ means the limit at $t_{n}$ of the argument of $G_{1}$ along the arc $\left[t_{n-1}, t_{n}\right]$ and $\arg \left(G_{1}\left(t_{n}+0\right)\right)$ means the limit at $t_{n}$ of the argument of $G_{1}$ along the arc $\left[t_{n}, t_{n+1}\right]$. The argument is taken to be continuous along each arc $\left[t_{n}, t_{n+1}\right]$.

In order to understand the monotonic behavior of $\arg G_{1}$ on the different arcs, and since $G_{1}$ is a product on $\sigma_{1}$ and $\sigma_{2}$, consider $w \in \overline{Q_{1}} \backslash\{-\bar{\omega}, i \omega\}$ and define

$$
\lambda_{1}(w)=\arg \left(\bar{\omega}^{2}-w^{2}\right) \quad \text { and } \quad \lambda_{2}(w)=\arg \left(\omega^{2}+w^{2}\right)=\arg \left(w^{2}-\left(-\omega^{2}\right)\right)
$$

note that $\lambda_{1}(w) \in\left(-\frac{\pi}{2}-2 \delta, \frac{\pi}{2}-2 \delta\right)$ and $\lambda_{2}(w) \in\left(-\frac{\pi}{2}+2 \delta, \frac{\pi}{2}+2 \delta\right)$.

Notice that if $w \in \sigma_{3}$, then $\lambda_{1}(w) \in\left[-\frac{\pi}{2}-\delta,-2 \delta\right)$ and if $w \in \sigma_{4}$, then $\lambda_{2}(w) \in\left(2 \delta, \frac{\pi}{2}+\delta\right]$. 
Let us factor $\bar{\omega}^{2}-w^{2}$ and $\omega^{2}+w^{2}$ and analyze these factors. Define $\alpha_{1}(w)=\arg (w-\bar{\omega}) \in$ $\left[\frac{3 \pi}{4}-\frac{\delta}{2}, \pi-\frac{\delta}{2}\right], \alpha_{2}(w)=\arg (w+i \omega) \in\left[\frac{\pi}{2}+\frac{\delta}{2}, \frac{3 \pi}{4}+\frac{\delta}{2}\right], \beta_{1}(w)=\arg (w+\bar{\omega})=\arg (w-(-\bar{\omega})) \in$ $\left(-\frac{\pi}{2}-\delta, \frac{\pi}{2}-\delta\right)$ and $\beta_{2}(w)=\arg (w-i \omega) \in(\delta-\pi, \delta)$. Then

$$
\lambda_{1}(w)=\alpha_{1}(w)+\beta_{1}(w)-\pi \quad \text { and } \quad \lambda_{2}(w)=\alpha_{2}(w)+\beta_{2}(w) .
$$

A few simple calculations show that if $w_{(1)}(\tau)=w_{1}+r_{1} e^{i \tau}$ parameterizes the circular arc $C_{1} \cap \overline{B_{1}}$, then

$$
\frac{d}{d \tau}\left(\alpha_{1}\left(w_{(1)}(\tau)\right)\right)<0, \quad \frac{d}{d \tau}\left(\beta_{1}\left(w_{(1)}(\tau)\right)\right)>0 \quad \text { and } \quad \frac{d}{d \tau}\left(\left(\alpha_{1}+\beta_{1}\right)\left(w_{(1)}(\tau)\right)\right)>0
$$

while if $w_{(2)}(\tau)=w_{2}+r_{2} e^{i \tau}$ parameterizes the circular arc $C_{2} \cap \overline{B_{1}}$, then

$$
\frac{d}{d \tau}\left(\alpha_{2}\left(w_{(2)}(\tau)\right)\right)<0, \quad \frac{d}{d \tau}\left(\beta_{2}\left(w_{(2)}(\tau)\right)\right)>0 \quad \text { and } \quad \frac{d}{d \tau}\left(\left(\alpha_{2}+\beta_{2}\right)\left(w_{(2)}(\tau)\right)\right)>0
$$

Hence $\lambda_{1}(w)$ is strictly increasing as $w \in \sigma_{1}$ moves in a clockwise direction with respect to $E$ (i.e. counterclockwise on $C_{1}$ ) and $\lambda_{2}(w)$ is strictly increasing as $w \in \sigma_{2}$ moves in a clockwise direction with respect to $E$ (i.e. counterclockwise on $C_{2}$ ). In a similar manner, notice that $\lambda_{1}(u)$ is strictly increasing for $u \in[-1,0]$ and $\lambda_{2}(i v)$ is strictly increasing for $v \in[0,1]$.

Now continuing our pursuit of the index of $G$, and following the methods of Lancaster and Athanassenas in [36], suppose that $\gamma_{1}+\gamma_{2} \geq \frac{\pi}{2}-2 \delta$, so that $k=3$. Then $t_{1}=w_{2}+r_{2} e^{i \tau_{2 A}}$ and $t_{3}=w_{2}+r_{2} e^{i \tau_{2 B}}$ for some $\tau_{2 A} \in\left[\gamma_{2}+\delta-\pi, 0\right)$ and $\tau_{2 B} \in\left(\delta-\frac{\pi}{2}, 0\right]$ with $\tau_{2 A}<\tau_{2 B}$. Further, $t_{1}=w_{1}+r_{1} e^{i \tau_{1 A}}$ and $t_{2}=w_{1}+r_{1} e^{i \tau_{1 B}}$ for some $\tau_{1 A} \in\left(-\frac{\pi}{2}, \frac{\pi}{2}-\delta-\gamma_{1}\right]$ and $\tau_{1 B} \in\left[-\frac{\pi}{2},-\delta\right)$ with $\tau_{1 A}>\tau_{1 B}$. We now see that $\alpha_{2}\left(t_{3}\right)=\arg \left(t_{3}+i \omega\right)<\frac{\pi}{2}+\delta, \beta_{2}\left(t_{3}\right)=\arg \left(t_{3}-i \omega\right)<\delta$, $\alpha_{1}\left(t_{2}\right)=\arg \left(t_{2}-\bar{\omega}\right)>\pi-\delta, \beta_{1}\left(t_{2}\right)=\arg \left(t_{2}+\bar{\omega}\right)>-\frac{\pi}{2}-\delta, \tau_{1 A}-\tau_{2 A}>0$ and (1.8) implies $\tau_{2 A}<0$ and $\tau_{1 A}>-\frac{\pi}{2}$. For the purpose of definiteness, set

$$
\begin{aligned}
& \arg \left(G_{1}\left(t_{1}-0\right)\right)=\pi+2 \delta-2\left(\tau_{2 A}+\lambda_{2}\left(t_{1}\right)\right) \\
& \arg \left(G_{1}\left(t_{1}+0\right)\right)=-2\left(\delta+\tau_{1 A}+\lambda_{1}\left(t_{1}\right)\right)
\end{aligned}
$$




$$
\begin{aligned}
& \arg \left(G_{1}\left(t_{2}-0\right)\right)=-2\left(\delta+\tau_{1 B}+\lambda_{1}\left(t_{2}\right)\right) \\
& \arg \left(G_{1}\left(t_{2}+0\right)\right)=\arg \left(G_{1}\left(t_{3}-0\right)\right)=\pi \\
& \arg \left(G_{1}\left(t_{3}+0\right)\right)=\pi+2 \delta-2\left(\tau_{2 B}+\lambda_{2}\left(t_{3}\right)\right) .
\end{aligned}
$$

Notice that

$$
\begin{gathered}
\theta_{1}=\frac{1}{2}+\frac{2 \delta+\tau_{1 A}-\tau_{2 A}+\lambda_{1}\left(t_{1}\right)-\lambda_{2}\left(t_{1}\right)}{\pi}, \\
\theta_{2}=-\frac{1}{2}-\frac{\delta+\lambda_{1}\left(t_{2}\right)+\tau_{1 B}}{\pi} \in\left(-\frac{1}{2}+\frac{2 \delta}{\pi}, \frac{1}{2}\right],
\end{gathered}
$$

and

$$
\theta_{3}=\frac{\lambda_{2}\left(t_{3}\right)+\tau_{2 B}-\delta}{\pi} \in\left(-\frac{1}{2}+\frac{2 \delta}{\pi}, \frac{1}{2}\right]
$$

Also

$$
\arg \left(G_{1}\left(t_{2}-0\right)\right)-\arg \left(G_{1}\left(t_{1}+0\right)\right)=2\left(\tau_{1 A}-\tau_{1 B}\right)+2\left(\lambda_{1}\left(t_{1}\right)-\lambda_{1}\left(t_{2}\right)\right)>0
$$

and

$$
\arg \left(G_{1}\left(t_{1}-0\right)\right)-\arg \left(G_{1}\left(t_{3}+0\right)\right)=2\left(\tau_{2 B}-\tau_{2 A}\right)+2\left(\lambda_{2}\left(t_{3}\right)-\lambda_{2}\left(t_{1}\right)\right)>0 ;
$$

hence $\theta_{1}+\theta_{2}+\theta_{3}>0$. Therefore, $\theta_{1}>1-\frac{4 \delta}{\pi}>0$.

Now $\tau_{1 A}-\tau_{2 A}$ equals the angle formed by the triangle with vertices $w_{1}, t_{1}$, and $w_{2}$ at the vertex $t_{1}$ and notice that $0<\tau_{1 A}-\tau_{2 A} \leq \pi$. Also, $\beta_{1}\left(t_{1}\right)-\beta_{2}\left(t_{1}\right)$ equals the angle at $t_{1}$ formed by the line segments $\left[t_{1},-\bar{\omega}\right]$ and $\left[t_{1}, i \omega\right]$ from $t_{1}$ to $-\bar{\omega}$ and $i \omega$ respectively, where the angle begins in the direction $\left[t_{1}, i \omega\right]$ and moves counterclockwise to $\left[t_{1},-\bar{\omega}\right]$. Notice that $\tau_{1 A}-\tau_{2 A}<\beta_{1}\left(t_{1}\right)-\beta_{2}\left(t_{1}\right)$ and $\frac{\pi}{4}-\delta<\beta_{1}\left(t_{1}\right)-\beta_{2}\left(t_{1}\right) \leq \frac{3 \pi}{4}+\delta$. Further, $\alpha_{1}\left(t_{1}\right)-\alpha_{2}\left(t_{1}\right)$ equals the (acute) angle formed by the triangle with vertices $-i \omega, t_{1}$, and $\bar{\omega}$ at the vertex $t_{1}$; notice that $\frac{\pi}{4}-\delta \leq \alpha_{1}\left(t_{1}\right)-\alpha_{2}\left(t_{1}\right)<\frac{\pi}{2}-2 \delta$.

Similarly to the conclusion of the previous paragraph, the authors observe that

$$
\theta_{1}>\frac{1}{2}+\frac{1}{\pi}\left(2 \delta+0+\left[\left(\frac{\pi}{4}-\delta\right)+\left(\frac{\pi}{4}-\delta\right)-\pi\right]\right)=0
$$

where the sum in the square brackets is a lower bound for $\lambda_{1}\left(t_{1}\right)-\lambda_{2}\left(t_{1}\right)$. In fact, as $\gamma_{1}$ and 
$\gamma_{2}$ converge to $\frac{\pi}{2}$ from below, $\theta_{2}$ and $\theta_{3}$ each converge to $-\frac{1}{2}+\frac{2 \delta}{\pi}$ and $\theta_{1}$ converges to $1-\frac{4 \delta}{\pi}$.

Setting $z_{1}=e^{i\left(\pi-\delta-\gamma_{1}\right)}$ and $z_{2}=e^{i\left(\frac{\pi}{2}+\delta+\gamma_{2}\right)}$, so that $z_{1} \in C_{1} \cap \partial B_{1}$ and $z_{2} \in C_{2} \cap \partial B_{1}$, then the monotonicity of $\lambda_{2}$ on $C_{2} \cap B_{1}$ implies

$$
\arg \left(G_{1}\left(t_{1}-0\right)\right) \leq \pi+2 \delta-2\left[\tau_{2 A}+\lambda_{2}\left(z_{2}\right)\right]=2 \pi-2 \tau_{2 A}-2 \delta-2 \gamma_{2}
$$

and the monotonicity of $\lambda_{1}$ on $C_{1} \cap B_{1}$ implies

$$
\arg \left(G_{1}\left(t_{1}+0\right)\right) \geq-2 \delta-2\left[\tau_{1 A}+\lambda_{1}\left(z_{1}\right)\right]=-\pi-2 \tau_{1 A}+2 \delta+2 \gamma_{1}
$$

Recalling that $\gamma_{1}+\gamma_{2} \geq \frac{\pi}{2}-2 \delta$, we obtain

$$
0<\theta_{1} \leq 1+\frac{\tau_{1 A}-\tau_{2 A}}{\pi} \leq 2
$$

and $\theta_{1}=2$ if and only if $\gamma_{1}+\gamma_{2}=\frac{\pi}{2}-2 \delta$.

Set $\nu_{1}=\left[\theta_{1}\right], \nu_{2}=0, \nu_{3}=0, \alpha_{1}=\theta_{1}-\nu_{1}, \alpha_{2}=\theta_{2}$ and $\alpha_{3}=\theta_{3}$, where $\left[\theta_{1}\right]$ denotes the greatest integer less than or equal to $\theta_{1}$. Notice that $\nu_{1}+\nu_{2}+\nu_{3} \in\{0,1,2\}$. Let $\psi$ be the conformal map from $E$ onto the unit disc $B_{1}$ which maps $t_{1}$ to $1, t_{2}$ to $i$ and $t_{3}$ to $-i$. Define $G^{*}: \partial B_{1} \rightarrow \mathbf{C}$ by

$$
G^{*}(\zeta)=G\left(\psi^{-1}(\zeta)\right) \quad \text { for } \zeta \in \partial B_{1}
$$

Our Hilbert problem (2.6) is then equivalent to finding $f^{*} \in C^{0}\left(\overline{B_{1}} \backslash\{1, \pm i\}\right)$ which is analytic in $B_{1}$, has (at worst) integrable singularities at $1, i,-i$, and satisfies

$$
\operatorname{Re}\left(G^{*}(\zeta) f^{*}(\zeta)\right)=0 \quad \text { for } \zeta \in \partial B_{1} \backslash\{1, \pm i\}
$$

Applying results (and notation) from Monakhov [44], Chapter 1, §4, 2. ${ }^{\circ}$ (a) (pp. 49-52) when $\nu_{1}=0$ after setting $\kappa=0$, (where $\kappa$ is the index of $G$ ) Monakhov [44], Chapter $1, \S 4$, 2. ${ }^{\circ}$ (b) (pp. 52-3) when $\nu_{1}=1$ after setting $\kappa=0$ and Monakhov [44], Chapter $1, \S 4,2 .^{\circ}$ (a) 
(pp. 49-52) when $\nu_{1}=2$ after setting $\kappa=1$, we obtain a solution $f=c f_{0}$ of (2.6), where $f_{0}$ has the form (for our example from [36])

$$
f_{0}(w)=(\psi(w)-1)^{\theta_{1}}(\psi(w)-i)^{\alpha_{2}}(\psi(w)+i)^{\alpha_{3}} e^{\Gamma_{0}(\psi(w))}
$$

\subsection{Computing A Capillary Surface}

In the general case, $f_{0}$ has the form

$$
f_{0}(w)=\left(w-t_{1}\right)^{\theta_{1}}\left(w-t_{2}\right)^{\theta_{2}} \ldots\left(w-t_{n}\right)^{\theta_{n}} e^{\Gamma_{0}(w)}
$$

and $\Gamma_{0}$ is defined as

$$
\Gamma_{0}=\frac{1}{2}\left\{\Gamma(z)+\Gamma_{*}(z)\right\}=\frac{1}{4 \pi i} \int_{|t|=1} \frac{\ln \left|\widetilde{G}_{0}(t)\right|}{t} \cdot \frac{t+w}{t-w} d t
$$

where

$$
G_{0}(t)=-t^{-2 \kappa} \prod_{k=1}^{n}\left(\frac{1}{t}-\overline{t_{k}}\right)^{\alpha_{k}}\left(t-t_{k}\right)^{-\alpha_{k}} \frac{\overline{G(t)}}{G(t)}
$$

and

$$
\widetilde{G}_{0}(t)= \begin{cases}G_{0}(t) & \text { when } \sum_{k=1}^{n} \nu_{k} \text { is even }\left(\kappa=\frac{1}{2} \sum_{k=1}^{n} \nu_{k}\right) \\ t^{-1} G_{0}(t) & \text { when } \sum_{k=1}^{n} \nu_{k} \text { is odd }\left(\kappa=\frac{1}{2}\left(\sum_{k=1}^{n} \nu_{k}-1\right)\right)\end{cases}
$$

Now, following a scheme from Henrici [21] (pages 108-111) for computing Cauchy Integrals on the disc, we find 


$$
\begin{aligned}
\Gamma_{0}\left(e^{2 i \pi \tau}\right) & =\frac{1}{4 i \pi} \int_{|t|=1} \frac{\ln \left|\widetilde{G}_{0}(t)\right|}{t} \cdot \frac{t+e^{2 i \pi \tau}}{t-e^{2 i \pi \tau}} d t \\
& =\frac{1}{4 i \pi} \int_{0}^{1} \frac{\ln \left|\widetilde{G}_{0}\left(e^{2 i \pi s}\right)\right|}{\cdot} \frac{e^{2 i \pi s}+e^{2 i \pi \tau}}{e^{2 i \pi s}-e^{2 i \pi \tau}} d s \\
& =\frac{1}{2} \int_{0}^{1} \ln \left|\widetilde{G}_{0}\left(e^{2 i \pi s}\right)\right|\left(1+\frac{2 e^{2 i \pi \tau}}{e^{2 i \pi s}-e^{2 i \pi \tau}}\right) d s \\
& =\Gamma_{0}(0)+e^{2 i \pi \tau} \int_{0}^{1} \ln \left|\widetilde{G}_{0}\left(e^{2 i \pi s}\right)\right| \frac{1}{e^{2 i \pi s}-e^{2 i \pi \tau}} d s \\
& =\Gamma_{0}(0)+e^{2 i \pi \tau} \int_{0}^{1} \ln \left|\widetilde{G}_{0}\left(e^{2 i \pi s}\right)\right| \frac{e^{-2 i \pi s}}{1-e^{2 i \pi(\tau-s)}} d s
\end{aligned}
$$

Now, for the Fourier coefficients $a_{k},(-\infty<k<\infty)$, set function $h\left(e^{2 i \pi s}\right)=e^{-2 i \pi s} \ln \left|\widetilde{G}_{0}\left(e^{2 i \pi s}\right)\right|$ such that $h\left(e^{2 i \pi s}\right)=\sum_{k=-\infty}^{\infty} a_{k} e^{2 i \pi s}$. Then

$$
\int_{0}^{1} h\left(e^{2 i \pi s}\right) \frac{e^{-2 i \pi s}}{1-e^{2 i \pi \tau} e^{-2 i \pi s}} d s=\frac{1}{2} a_{0}+\frac{1}{2} \sum_{k=1}^{\infty}\left(a_{k} e^{2 i \pi k \tau}+a_{-k} a_{k} e^{-2 i \pi k \tau}\right.
$$

and so

$$
\Gamma_{0}\left(e^{2 i \pi \tau}\right)=\Gamma_{0}(0)+\frac{1}{2} a_{0} e^{2 i \pi \tau}+\frac{1}{2} \sum_{k=1}^{\infty}\left(a_{k} e^{2 i \pi(k+1) \tau}+a_{-k} a_{k} e^{-2 i \pi(k-1) \tau}\right) .
$$

To implement this numerically, Henrici chooses a vector $N$ of equally spaced data points around the unit circle, preferably equal to $2^{n}$ for $n$ large and defines the finite sequence $h_{k}$, for $k \in \mathbb{Z}$ as $h_{k}=h\left(e^{2 i \pi \frac{k}{N}}\right)$.

Next, Henrici notes that by applying a Fast Fourier Transform (FFT) the discrete Fourier coefficients of $h_{k}(k \in(-N, N))$, with these coefficients denoted by sequence $c=\left\{c_{k}\right\}$ where $c=\mathcal{F}_{n}(h)$ and $|k| \leq 2^{n-1}$, can be computed in $O(n \operatorname{Logn})$ operations using only one application of FFT. Now the $c_{j}$ are reasonable approximations for the $a_{j}$ for $|k| \leq m=2^{n-1}$ 
and so our approximation of $\Gamma_{0}(w)$ for $w \in \mathbb{C}$ is

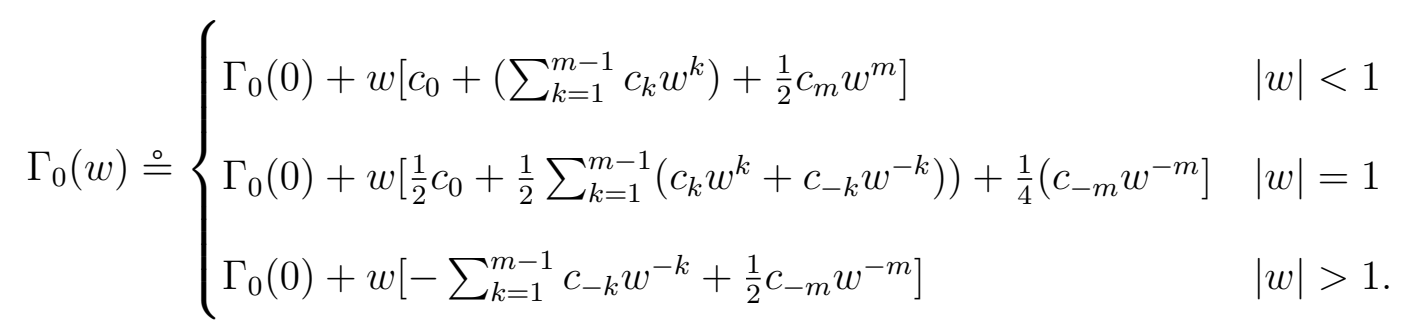

Now that we have an estimate for $\Gamma_{0}$, we can compute $f_{0}$ and therefore $f=c f_{0}$ for some appropriate scaling constant $c$. This $f$ is the function $f$ from the Enneper-Weierstrass (f,g) representation.

All that is needed now to compute a capillary surface $X$ is the function $g$ and EnneperWeierstrass can be applied to compute the surface. However, $g$ is really a conformal map of the set $E$ from $§ 1.4$. We will discuss our attempt at conformal mapping in the next section.

To calculate surface $X=(x(w), y(w), z(w))$, an application of Enneper-Weierstrass gives

$$
\begin{aligned}
& x(w)=x_{0}+\operatorname{Re}\left\{\frac{1}{2} \int_{0}^{w} f(t)\left(1-g^{2}(t)\right) d t\right\} \\
& y(w)=y_{0}+\operatorname{Re}\left\{\frac{i}{2} \int_{0}^{w} f(t)\left(1+g^{2}(t)\right) d t\right\} \\
& z(w)=z_{0}+\operatorname{Re}\left\{\int_{0}^{w} f(t) g(t) d t\right\} .
\end{aligned}
$$

To compute these integrals numerically, we recommend Simpson's Rule.

\subsection{Conformal Mapping}

Numerical investigation of capillary graphs include conformally mapping the stereographic projection of the Gauss map to the unit disk. When the stereographic projection is a polygon we recommend Tobin Driscoll's excellent Schwarz-Christoffel Toolbox for MATLAB ${ }^{\mathrm{TM}}$, (c)1998. Professor Driscoll created several object-oriented classes to provide methods for investigating conformal maps to both the upper half plane and the unit disk. 
These classes provide forward and inverse maps as well a a rich set of underlying value classes such as the polygon class. The toolbox classes attain an accuracy on the order of $10^{-14}$ when used for a polygon with such a small number of vertices as these projections.

However the projection of the Gauss map of a non-convex corner is a circular arc polygon as in figure 1.6 in the continuous case, and figure 1.7 in the discontinuous case. Numerical investigation utilizing polygonal approximation of smooth curve boundaries gives mixed results. There exists no proof ensuring solution of the 'parameter problem' for polygons with enough vertices to approximate a circular polygon segment. The parameter problem consists of determining pre-vertices on the boundary of the unit disk that give an accurate conformal map. There are also serious questions about the accuracy of a polygonal approximation to a non-convex corner due to the so-called 'crowding phenomenon'.

In light of these issues we also currently investigate the zipper algorithm. Like the SchwarzChristoffel Toolbox, the zipper algorithm promises to be fast and accurate, and convergence has been proven, in the sense of uniformly close boundaries, for Jordan regions whose boundary lie on a $\mathrm{C}^{1}$ curve( [40]). Unlike the SchwarzChristoffel Toolbox, there is only one example program available, written by Marshall in Fortran, that implements the zipper algorithm. There are limitations to the sample program that exclude circular arc polygons such as figure 1.6 and figure 1.7. In Marshall's recent work he describes a modification of the geodesic algorithm for more accurate numerical computation and to "avoid errors ... as several people have encountered when programming the algorithm."( [41]) We too have encountered several issues, that we continue to resolve, since existing implementations make simplifying assumptions inconsistent with our requirements. Our ongoing investigation concerns implementing the zipper algorithm in a set of object-oriented MATLAB ${ }^{\mathrm{TM}}$ classes as in Driscoll's work.

In order to describe these issues we need to review a few details of the zipper algorithm implementation. The heart of the zipper algorithm consists of discretizing the boundary of a Jordan region whose boundary lies on a $\mathrm{C}^{1}$ curve and incrementally mapping the arc 
described by consecutive triplets of the discretization to the boundary of the right half-plane via a sequence of conformal maps. If the arc $\sigma$ described by each consecutive three points 0 , $\eta$ and $\zeta$ of the discretization of the boundary is orthogonal to the imaginary axis, we then map the hyperbolic geodesic to the imaginary axis via a Möbius transformation composed with the map $S(z)=\sqrt{z^{2}-1}$. Using the right half plane, $\mathbb{H}^{+}$, ensures that we remain on the correct branch. The Möbius transformation is given by

$$
L_{\sigma}=\frac{c z}{1+i d z}
$$

where $\zeta=a+i b \in \mathbb{H}^{+}, c=a /\left(a^{2}+b^{2}\right)$ and $d=b /\left(a^{2}+b^{2}\right)$. The composition $h_{\sigma}=\sqrt{L_{\sigma}^{2}-1}$ is a conformal map of $\mathbb{H}^{+} \backslash \sigma$ onto $\mathbb{H}^{+}$.

If, as usually occurs, $\sigma$ is not orthogonal to the imaginary axis, then the sequence of maps consists of a Möbius transformation $\sigma$ to a straight line segment followed by the inverse of a ślit map'. The Möbius transformation is given by

$$
\ell_{\sigma}=\frac{z}{1-z / \delta}
$$

where $\delta$ is the intersection of the circle containing $\sigma$ with the imaginary axis other than 0 , and $\zeta^{\prime}=\ell_{\sigma}(\delta)$. The slit map discovered by Marshall ( [40]) is a conformal map, the Möbius transformation of the arc to a straight line segment $g_{\zeta^{\prime}}: \mathbb{H}^{+} \rightarrow \mathbb{H}^{+} \backslash L$, where $L$ is a line segment from 0 to $\zeta^{\prime}$. Explicitly

$$
g_{\zeta^{\prime}}(z)=\left|\zeta^{\prime}\right| \frac{(i z-p)^{p}(i z+1-p)^{1-p}}{p^{p}(1-p)^{1-p}},
$$

where $p=\arg \zeta^{\prime} / \pi \in(0,2)$. Hence our conformal map is given by $h_{\sigma}=g_{\zeta^{\prime}}^{-1} \circ \ell_{\sigma}$. We encountered several difficulties that must be overcome over the next few months. These are among the items discussed in Nguyen's Thesis [47]:

- Marshall's most recent paper [41] improves the geodesic algorithm. Some of these 
improvements include modifying computations made in the first paper and existing code from maps onto the upper half plane to $\mathbb{H}^{+}$, the right half-plane. However he leaves improving and re-writing the remaining maps to the reader.

- Since the inverse of the slit map can not be described by simple functions, one must numerically approximate the inverse slit map. Existing code makes simplifying assumptions in order to compute such an approximation and these assumptions exclude circular arc polygons such as figure 1.6 and figure 1.7. We need a robust approximation to $g_{\zeta^{\prime}}$ that does not require, for example, the assumption that no circular arc segments meet at a vertex of the circular arc polygon, or that the convex region of all circular arc segments are disjoint from the convex hull of the vertices of the polygon, as Zipper encounters difficulties at these points (for example, taking the wrong branch of the square root or sending points to infinity). 
Chapter 3

\section{A Capillary Surface with No Radial Limits}

\subsection{Introduction}

Recalling our domain, let $\Omega$ be a domain in $\mathbb{R}^{2}$ with locally Lipschitz boundary and $O=(0,0) \in \partial \Omega$ such that $\partial \Omega \backslash\{O\}$ is a $C^{4}$ curve and $\Omega \subset B_{1}(0,1)$, where $B_{\delta}(N)$ is the open ball in $\mathbb{R}^{2}$ of radius $\delta$ about $N \in \mathbb{R}^{2}$. Denote the unit exterior normal to $\Omega$ at $(x, y) \in \partial \Omega$ by $\nu(x, y)$ and let polar coordinates relative to $O$ be denoted by $r$ and $\theta$. We shall assume there exists a $\delta^{*} \in(0,2)$ and $\alpha \in\left(0, \frac{\pi}{2}\right)$ such that $\partial \Omega \cap B_{\delta^{*}}(O)$ consists of the line segments

$$
\partial^{+} \Omega^{*}=\left\{(r \cos (\alpha), r \sin (\alpha)): 0 \leq r \leq \delta^{*}\right\}
$$

and

$$
\partial^{-} \Omega^{*}=\left\{(r \cos (-\alpha), r \sin (-\alpha)): 0 \leq r \leq \delta^{*}\right\}
$$

Set $\Omega^{*}=\Omega \cap B_{\delta^{*}}(O)$. Let $\gamma: \partial \Omega \rightarrow[0, \pi]$ be given. Let $\left(x^{ \pm}(s), y^{ \pm}(s)\right)$ be arclength parametrizations of $\partial^{ \pm} \Omega$ with $\left(x^{+}(0), y^{+}(0)\right)=\left(x^{-}(0), y^{-}(0)\right)=(0,0)$ and set $\gamma^{ \pm}(s)=$ $\gamma\left(x^{ \pm}(s), y^{ \pm}(s)\right)$

Consider the capillary problem of finding a function $f \in C^{2}(\Omega) \cap C^{1}(\bar{\Omega} \backslash\{O\})$ satisfying

$$
\operatorname{div}(T f)=\frac{1}{2} f \text { in } \Omega
$$

and

$$
T f \cdot \nu=\cos (\gamma) \text { on } \partial \Omega \backslash\{O\}
$$

where $T f=\frac{\nabla f}{\sqrt{1+|\nabla f|^{2}}}$. We are interested in the existence of the radial limits $R f(\cdot)$ of a 
solution $f$ of $(3.1)-(3.2)$, where

$$
R f(\theta)=\lim _{r \rightarrow 0^{+}} f(r \cos \theta, r \sin \theta),-\alpha<\theta<\alpha
$$

and $R f( \pm \alpha)=\lim _{\partial^{ \pm} \Omega^{*} \ni \mathbf{x} \rightarrow O} f(\mathbf{x}), \mathbf{x}=(x, y)$, which are the limits of the boundary values of $f$ on the two sides of the corner if these exist. In [10], the following is proven:

Proposition 2. Let $f$ be a bounded solution to (3.1) satisfying (3.2) on $\partial^{ \pm} \Omega^{*} \backslash\{O\}$ which is discontinuous at $O$. If $\alpha>\pi / 2$ then $R f(\theta)$ exists for all $\theta \in(-\alpha, \alpha)$. If $\alpha \leq \pi / 2$ and there exist constants $\underline{\gamma}^{ \pm}, \bar{\gamma}^{ \pm}, 0 \leq \underline{\gamma}^{ \pm} \leq \bar{\gamma}^{ \pm} \leq \pi$, satisfying

$$
\pi-2 \alpha<\underline{\gamma}^{+}+\underline{\gamma}^{-} \leq \bar{\gamma}^{+}+\bar{\gamma}^{-}<\pi+2 \alpha
$$

so that $\underline{\gamma}^{ \pm} \leq \gamma^{ \pm}(s) \leq \bar{\gamma}^{ \pm}$for all $s, 0<s<s_{0}$, for some $s_{0}$, then again $R f(\theta)$ exists for all $\theta \in(-\alpha, \alpha)$.

In [37], Lancaster and Siegel proved this theorem with the additional restriction that $\gamma$ be bounded away from 0 and $\pi$; Figure 3.1 illustrates these cases.

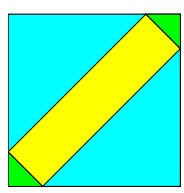

A

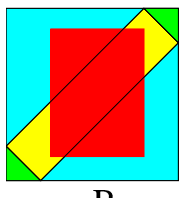

B
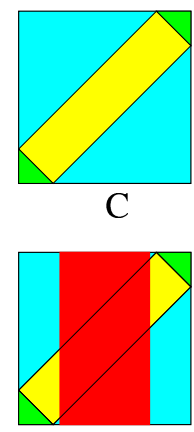

$\mathrm{D}$

Figure 3.1: The Concus-Finn rectangle (A \& C) with regions $R$ (yellow), $D_{2}^{ \pm}$(blue) and $D_{1}^{ \pm}$ (green); the restrictions on $\gamma$ in [37] (red region in B) and in [10] (red region in D).

In Theorem 3 of [37], Lancaster and Siegel also proved

Proposition 3. Let $\Omega$ be the disk of radius 1 centered at $(1,0)$. Then there exists a solution to $N f=\frac{1}{2} f$ in $\Omega,|f| \leq 2, f \in C^{2}(\Omega) \cap C^{1}(\bar{\Omega} \backslash O), O=(0,0)$ so that no radial limits $R f(\theta)$ 
exist $(\theta \in[-\pi / 2, \pi / 2])$.

In this case, $\alpha=\frac{\pi}{2}$; if $\gamma$ is bounded away from 0 and $\pi$, then Proposition 2 would imply that $R f(\theta)$ exists for each $\theta \in\left[-\frac{\pi}{2}, \frac{\pi}{2}\right]$ and therefore the contact angle $\gamma=\cos ^{-1}(T f \cdot \nu)$ in Proposition 3 is not bounded away from 0 and $\pi$.

\subsection{A Surface Defined by Contact Angle Data}

In our case, the domain $\Omega$ has a convex corner of size $2 \alpha$ at $O$ and we wish to investigate the question of whether an example like that in Proposition 3 exists in this case when $\gamma$ is bounded away from 0 and $\pi$. In terms of the Concus-Finn rectangle, the question is whether, given $\epsilon>0$, there is a $f \in C^{2}(\Omega) \cap C^{1}(\bar{\Omega} \backslash\{O\})$ of (3.1)-(3.2) such that no radial limits $R f(\theta)$ exist $(\theta \in[-\alpha, \alpha])$ and $\left|\gamma-\frac{\pi}{2}\right| \leq \alpha+\epsilon$; this is illustrated in Figure 3.2.
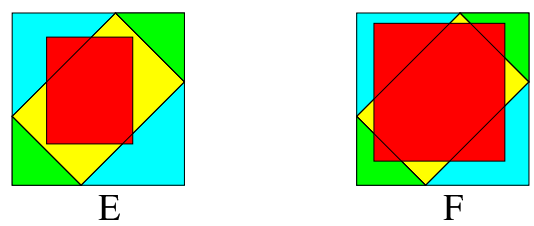

Figure 3.2: The Concus-Finn rectangle. When $\gamma$ remains in red region in E, $R f(\cdot)$ exists; $\gamma$ in Theorem 3 remains in the red region in $\mathrm{F}$.

Theorem 3. For each $\epsilon>0$, there is a domain $\Omega$ as described above and a solution $f \in$ $C^{2}(\Omega) \cap C^{1}(\bar{\Omega} \backslash\{O\})$ of (3.1) such that the contact angle $\gamma=\cos ^{-1}(T f \cdot \nu): \partial \Omega \backslash\{O\} \rightarrow[0, \pi]$ satisfies $\left|\gamma-\frac{\pi}{2}\right| \leq \alpha+\epsilon$ and there exist a sequence $\left\{r_{j}\right\}$ in $(0,1)$ with $\lim _{j \rightarrow \infty} r_{j}=0$ such that

$$
(-1)^{j} f\left(r_{j}, 0\right)>1 \quad \text { for each } j \in \mathbb{N} \text {. }
$$

Assuming $\Omega$ and $\gamma$ are symmetric with respect to the line $\{(x, 0): x \in \mathbb{R}\}$, this implies that no radial limit

$$
R f(\theta) \stackrel{\text { def }}{=} \lim _{r \downarrow 0} f(r \cos (\theta), r \sin (\theta))
$$

exists for any $\theta \in[-\alpha, \alpha]$. 
We note that our Theorem is an extension of Theorem 3 of [37] to contact angle data in a domain with a convex corner. As in $[32,37]$, we first state and prove a localization lemma; this is analogous to the Lemma in [32] and Lemma 2 of [37].

Lemma 1. Let $\Omega \subseteq \mathbb{R}^{2}$ be as above, $\epsilon>0, \eta>0$ and $\gamma_{0}: \partial \Omega \backslash\{O\} \rightarrow[0, \pi]$ such that $\left|\gamma_{0}-\frac{\pi}{2}\right| \leq \alpha+\epsilon$. For each $\delta \in(0,1)$ and $h \in C^{2}(\Omega) \cap C^{1}(\bar{\Omega} \backslash\{O\})$ which satisfies (3.1) and (3.2) with $\gamma=\gamma_{0}$, there exists a solution $g \in C^{2}(\Omega) \cap C^{1}(\bar{\Omega} \backslash\{O\})$ of (3.1) such that $\lim _{\bar{\Omega} \ni(x, y) \rightarrow(0,0)} g(x, y)=+\infty$,

$$
\sup _{\Omega_{\delta}}|g-h|<\eta \quad \text { and } \quad\left|\gamma_{g}-\frac{\pi}{2}\right| \leq \alpha+\epsilon
$$

where $\Omega_{\delta}=\bar{\Omega} \backslash B_{\delta}(O)$ and $\gamma_{g}=\cos ^{-1}(T g \cdot \nu): \partial \Omega \backslash\{O\} \rightarrow[0, \pi]$ is the contact angle which the graph of $g$ makes with $\partial \Omega \times \mathbb{R}$.

Proof. Let $\epsilon, \eta, \delta, \Omega, h$ and $\gamma_{0}$ be given. For $\beta \in(0, \delta)$, let $g_{\beta} \in C^{2}(\Omega) \cap C^{1}(\bar{\Omega} \backslash\{O\})$ satisfy (3.1) and (3.2) with $\gamma=\gamma_{\beta}$, where

$$
\gamma_{\beta}=\left\{\begin{array}{cccc}
\frac{\pi}{2}-\alpha-\epsilon & \text { on } & \overline{B_{\beta}(O)} \\
\gamma_{0} & \text { on } & \bar{\Omega} \backslash B_{\beta}(O)
\end{array}\right.
$$

As in the proof of Theorem 3 of [37], $g_{\beta}$ converges to $h$, pointwise and uniformly in the $C^{1}$ norm on $\overline{\Omega_{\delta}}$ as $\beta$ tends to zero. Fix $\beta>0$ small enough that $\sup _{\Omega_{\delta}}|g-h|<\eta$.

Set $\Sigma=\{(r \cos (\theta), r \sin (\theta)): r>0,-\alpha \leq \theta \leq \alpha\}$. Now define $w: \Sigma \rightarrow \mathbb{R}$ by

$$
w(r \cos \theta, r \sin \theta)=\frac{\cos \theta-\sqrt{k^{2}-\sin ^{2} \theta}}{k \kappa r}
$$

where $k=\sin \alpha \sec \left(\frac{\pi}{2}-\alpha-\epsilon\right)=\sin \alpha \csc (\alpha+\epsilon)$. As in [4], there exists a $\delta_{1}>0$ such that $\operatorname{div}(T w)-\frac{1}{2} w \geq 0$ on $\Sigma \cap B_{\delta_{1}}(O), T w \cdot \nu=\cos \left(\frac{\pi}{2}-\alpha-\epsilon\right)$ on $\partial \Sigma \cap B_{\delta_{1}}(O)$, and 
$\lim _{r \rightarrow 0^{+}} w(r \cos \theta, r \sin \theta)=\infty$ for each $\theta \in[-\alpha, \alpha]$. We may assume $\delta_{1} \leq \delta^{*}$. Let

$$
M=\sup _{\Omega \cap \partial B_{\delta_{1}}(O)}\left|w-g_{\beta}\right| \text { and } w_{\beta}=w-M .
$$

Since $\operatorname{div}\left(T w_{\beta}\right)-\frac{1}{2} w_{\beta} \geq \frac{M}{2} \geq 0=\operatorname{div}\left(T g_{\beta}\right)-\frac{1}{2} g_{\beta}$ in $\Omega \cap B_{\delta_{1}}(O), w_{\beta} \leq g_{\beta}$ on $\Omega \cap \partial B_{\delta_{1}}(O)$ and $T g_{\beta} \cdot \nu \geq T w_{\beta} \cdot \nu$ on $\partial \Omega \cap B_{\delta_{1}}(O)$, we see that $g_{\beta} \geq w_{\beta}$ on $\Omega \cap \partial B_{\delta_{1}}(O)$.

We may now prove Theorem 3.

Proof. We shall construct a sequence $f_{n}$ of solutions of (3.1) and a sequence $\left\{r_{n}\right\}$ of positive real numbers such that $\lim _{n \rightarrow \infty} r_{n}=0, f_{n}(x, y)$ is even in $y$ and

$$
(-1)^{j} f_{n}\left(r_{j}, 0\right)>1 \quad \text { for each } j=1, \ldots, n
$$

Let $\gamma_{0}=\frac{\pi}{2}$ and $f_{0}=0$. Set $\eta_{1}=1$ and $\delta_{1}=\delta_{0}$. From Lemma 1 , there exists a $f_{1} \in$ $C^{2}(\Omega) \cap C^{1}(\bar{\Omega} \backslash\{O\})$ which satisfies (3.1) such that $\sup _{\Omega_{\delta_{1}}}\left|f_{1}-f_{0}\right|<\eta_{1},\left|\gamma_{1}-\frac{\pi}{2}\right| \leq \alpha+\epsilon$ and $\lim _{\Omega \ni(x, y) \rightarrow O} f_{1}(x, y)=-\infty$, where $\gamma_{1}=\cos ^{-1}\left(T f_{1} \cdot \nu\right)$. Then there exists $r_{1} \in\left(0, \delta_{1}\right)$ such that $f_{1}\left(r_{1}, 0\right)<-1$.

Now set $\eta_{2}=-\left(f_{1}\left(r_{1}, 0\right)+1\right)>0$ and $\delta_{2}=r_{1}$. From Lemma 1 , there exists a $f_{2} \in$ $C^{2}(\Omega) \cap C^{1}(\bar{\Omega} \backslash\{O\})$ which satisfies (3.1) such that $\sup _{\Omega_{\delta_{2}}}\left|f_{2}-f_{1}\right|<\eta_{2},\left|\gamma_{2}-\frac{\pi}{2}\right| \leq \alpha+\epsilon$ and $\lim _{\Omega \ni(x, y) \rightarrow O} f_{2}(x, y)=\infty$, where $\gamma_{2}=\cos ^{-1}\left(T f_{2} \cdot \nu\right)$. Then there exists $r_{2} \in\left(0, \delta_{2}\right)$ such that $f_{2}\left(r_{2}, 0\right)>1$. Since $\left(r_{1}, 0\right) \in \Omega_{\delta_{2}}$,

$$
f_{1}\left(r_{1}, 0\right)+1<f_{2}\left(r_{1}, 0\right)-f_{1}\left(r_{1}, 0\right)<-\left(f_{1}\left(r_{1}, 0\right)+1\right)
$$

and so $f_{2}\left(r_{1}, 0\right)<-1$.

Next set $\eta_{3}=\min \left\{-\left(f_{2}\left(r_{1}, 0\right)+1\right), f_{2}\left(r_{2}, 0\right)-1\right\}>0$ and $\delta_{3}=r_{2}$. From Lemma 1, there exists a $f_{3} \in C^{2}(\Omega) \cap C^{1}(\bar{\Omega} \backslash\{O\})$ which satisfies (3.1) such that $\sup _{\Omega_{\delta_{3}}}\left|f_{3}-f_{2}\right|<\eta_{3}$, $\left|\gamma_{3}-\frac{\pi}{2}\right| \leq \alpha+\epsilon$ and $\lim _{\Omega \ni(x, y) \rightarrow O} f_{3}(x, y)=-\infty$, where $\gamma_{3}=\cos ^{-1}\left(T f_{3} \cdot \nu\right)$. Then there 
exists $r_{3} \in\left(0, \delta_{3}\right)$ such that $f_{3}\left(r_{3}, 0\right)<-1$. Since $\left(r_{1}, 0\right),\left(r_{2}, 0\right) \in \Omega_{\delta_{2}}$, we have

$$
f_{2}\left(r_{1}, 0\right)+1<f_{3}\left(r_{1}, 0\right)-f_{2}\left(r_{1}, 0\right)<-\left(f_{2}\left(r_{1}, 0\right)+1\right)
$$

and

$$
-\left(f_{2}\left(r_{2}, 0\right)-1\right)<f_{3}\left(r_{2}, 0\right)-f_{2}\left(r_{2}, 0\right)<f_{2}\left(r_{2}, 0\right)-1
$$

hence $f_{3}\left(r_{1}, 0\right)<-1$ and $1<f_{3}\left(r_{2}, 0\right)$.

Continuing to define $f_{n}$ and $r_{n}$ inductively, we set

$$
\eta_{n+1}=\min _{1 \leq j \leq n}\left|f_{n}\left(r_{j}, 0\right)-(-1)^{j}\right| \quad \text { and } \quad \delta_{n+1}=\min \left\{r_{n}, \frac{1}{n}\right\}
$$

From Lemma 1, there exists $f_{n+1} \in C^{2}(\Omega) \cap C^{1}(\bar{\Omega} \backslash\{O\})$ which satisfies (3.1) such that $\sup _{\Omega_{\delta_{n+1}}}\left|f_{n+1}-f_{n}\right|<\eta_{n+1},\left|\gamma_{n+1}-\frac{\pi}{2}\right| \leq \alpha+\epsilon$ and $\lim _{\Omega \ni(x, y) \rightarrow O} f_{n+1}(x, y)=(-1)^{n+1} \infty$, where $\gamma_{n+1}=\cos ^{-1}\left(T f_{n+1} \cdot \nu\right)$. Then there exists $r_{n+1} \in\left(0, \delta_{n+1}\right)$ such that $(-1)^{n+1} f_{n+1}\left(r_{n+1}, 0\right)>$ 1. For each $j \in\{1, \ldots, n\}$ which is an even number, we have

$$
-\left(f_{n}\left(r_{j}, 0\right)-1\right)<f_{n+1}\left(r_{j}, 0\right)-f_{n}\left(r_{j}, 0\right)<f_{n}\left(r_{j}, 0\right)-1
$$

and so $1<f_{n+1}\left(r_{j}, 0\right)$. For each $j \in\{1, \ldots, n\}$ which is an odd number, we have

$$
f_{n}\left(r_{j}, 0\right)+1<f_{n+1}\left(r_{j}, 0\right)-f_{n}\left(r_{j}, 0\right)<-\left(f_{n}\left(r_{j}, 0\right)+1\right)
$$

and so $f_{n+1}\left(r_{j}, 0\right)<-1$.

As in $[37,52]$, there is a subsequence of $\left\{f_{n}\right\}$, still denoted $\left\{f_{n}\right\}$, which converges pointwise and uniformly in the $C^{1}$ norm on $\overline{\Omega_{\delta}}$ for each $\delta>0$ as $n \rightarrow \infty$ to a solution $f \in C^{2}(\Omega) \cap$ $C^{1}(\bar{\Omega} \backslash O)$ of (3.1). For each $j \in \mathbb{N}$ which is even, $f_{n}\left(r_{j}, 0\right)>1$ for each $n \in \mathbb{N}$ and so $f\left(r_{j}, 0\right) \geq 1$. For each $j \in \mathbb{N}$ which is odd, $f_{n}\left(r_{j}, 0\right)<-1$ for each $n \in \mathbb{N}$ and so 
$f\left(r_{j}, 0\right) \leq-1$. Therefore

$$
\lim _{r \rightarrow 0^{+}} f(r, 0) \text { does not exist, even as an infinite limit, }
$$

and so $R f(0)$ does not exist.

Since $\Omega$ is symmetric with respect to the $x$-axis and $\gamma_{n}(x, y)$ is an even function of $y$, $f(x, y)$ is an even function of $y$. Now suppose that there exists $\theta_{0} \in[-\alpha, \alpha]$ such that $R f\left(\theta_{0}\right)$ exists; then $\theta_{0} \neq 0$. From the symmetry of $f, R f\left(-\theta_{0}\right)$ must also exist and $R f\left(-\theta_{0}\right)=R f\left(\theta_{0}\right)$. Set $\Omega^{\prime}=\left\{(r \cos \theta, r \sin \theta): 0<r<\delta_{0},-\theta_{0}<\theta<\theta_{0}\right\} \subset \Omega$. Since $f$ has continuous boundary values on $\partial \Omega^{\prime}, f \in C^{0}\left(\overline{\Omega^{\prime}}\right)$ and so $R f(0)$ does exist, which is a contradiction. Thus $R f(\theta)$ does not exist for any $\theta \in[-\alpha, \alpha]$.

\subsection{Concluding Remarks}

Much of the previous research in Capillary Surfaces with Radial Limits and the Central Fan Conjecture is reliant on Dirichlet Data for computations and development of said theory. As is well known, Dirichlet data implies that the surface has no velocity with respect to the boundary and so is time independent. With this work we hope to begin to widen the extant research to encompass Contact Angle data as well, thereby perhaps offering research opportunities for time dependent Capillary inquiries with the goal of better understandings the Capillary Problem from an engineering and/or physics standpoint. One of the goals of which is assisting in understanding the requirements for fluid containment and condensation dissipation for deep space missions. 
REFERENCES 


\section{REFERENCES}

[1] L. V. Ahlfors, Complex Analysis, Third Edition, McGraw-Hill, New York, 1979.

[2] J. N. Crenshaw, A. K. Echart and K. E. Lancaster, A Generalization of "Existence and Behavior of the Radial Limits of a Bounded Capillary Surface at a Corner", submitted to the Pacific J. Math. preprint. arXiv:1701.03336

[3] P. Concus and R. Finn, On the behavior of a capillary surface in a wedge, Proceeding of the National Acedemy of Science, 63 (1969), 292-299.

[4] P. Concus and R. Finn, On a Class Of Capillary Surfaces, Journal d'Analyse Mathmatique Vol. 23 No. 1 (1970), 65-70. Acta Math. 132 (1974), 177-198.

[5] P. Concus and R. Finn, On capillary free surfaces in the absence of gravity, Acta Math. 132 (1974), 177-198.

[6] P. Concus and R. Finn, On capillary free surfaces in a gravitational field, Acta Math. 132 (1974), 207-223.

[7] P. Concus and R. Finn, Capillary wedges revisited., SIAM J. Math. Anal. 27 , 1 (1996), $56-69$.

[8] R. Courant, Dirichlet's Principle, Conformal Mapping, and Minimal Surfaces, Interscience, New York, 1950.

[9] J. T. Chen, R. Finn and E. Miersemann, Capillary surfaces in wedge domains, Pacific J. Math. 236 (2008), no. 2, 283-305.

[10] J. N. Crenshaw, A. K. Echart and K. E. Lancaster, A Generalization of "Existence and Behavior of the Radial Limits of a Bounded Capillary Surface at a Corner", submitted to the Pacific J. Math. arXiv:1701.03336

[11] J. Crenshaw and K. E. Lancaster, Behavior of some CMC capillary surfaces at convex corners., Pacific J. Math. 224 (2006), no. 2, 231-246.

[12] A. Elcrat and K. E. Lancaster, On the behavior of a nonparametric minimal surface in a nonconvex quadrilateral., Arch. Rational Mech. Anal. 94 , 3 (1986), 209-226.

[13] A. Elcrat and K. E. Lancaster, Boundary behavior of a nonparametric surface of prescribed mean curvature near a reentrant corner, Trans. Amer. Math. Soc. 297 (1986), 645-650.

[14] A. Elcrat and K. E. Lancaster, Bernstein functions and the Dirichlet problem., SIAM J. Math. Anal., 5 (1989), 1055-1068.

[15] M. Emmer, Esistenza, unicit e regolarit nelle superfici de equilibrio nei capillari, Ann. Univ. Ferrara Sez. VII (N.S.) 18 (1973), 79-94.

[16] R. Finn, Equilibrium Capillary Surfaces, Springer-Verlag, New York, 1986. 
[17] R. Finn, Local and global criteria for capillary surfaces in wedges, Calc. Var. Partial Differential Equations 4 (1996), no. 4, 305-322.

[18] R. Finn, Featured Review of "Existence and behavior of the radial limits of a bounded capillary surface at a corner" by Lancaster and Siegel, Math Reviews MR1433987 (98g:58030a) and MR1452541 (98g:58030b).

[19] R. Finn and C. Gerhardt, The internal sphere condition and the capillary problem, Ann. Mat. Pura Appl. (4) 112 (1977), 13-31.

[20] J. B. Garnett and D. E. Marshall, Harmonic Measure, Cambridge University Press, New York, 2005.

[21] P. Henrici, Applied and Computational Complex Analysis, Volume 3, Wiley-Interscience, New York, NY, 1993.

[22] R. Huff and J. McCuan, Scherk-Type Capillary Graphs, J. Math. Fluid Mech. 8 (2006), no. $1,99-119$.

[23] T. Jeffres and K. Lancaster, Vertical blow ups of capillary surfaces in $\mathbb{R}^{3}$. I. Convex corners, Electron. J. Differential Equations 2007, No. 152, 24 pp.

[24] T. Jeffres and K. Lancaster, Vertical Blow Ups of Capillary Surfaces in $\mathbb{R}^{3}$ Part 2: Nonconvex Corners, Electron. J. Differential Equations 2008, No. 160, 25 pp.

[25] Z. Jin and K. Lancaster, Theorems of Phragmn-Lindelf type for quasilinear elliptic equations, J. Reine Angew. Math. 514 (1999), 165-197.

[26] K. Kenmotsu, Surfaces with Constant Mean Curvature, Translations of Mathematical Monographs, 221. American Mathematical Society, Providence, RI, 2003.

[27] N. J. Korevaar, On the behavior of a capillary surface at a re-entrant corner, Pacific J. Math. 88 (1980), 379-385.

[28] N. J. Korevaar, Capillary surface convexity above convex domains., Indiana Univ. Math. J. 32,1 (1983), 73-81.

[29] N. J. Korevaar, Capillary surface regularity in corner subdomains of $\mathbb{R}^{n}$, Miniconference on geometry and partial differential equations, Proc. Centre Math. Anal. Austral. Nat. Univ., 12, Austral. Nat. Univ., Canberra, (1987), 67-71.

[30] K. E. Lancaster, Boundary behavior of a non-parametric minimal surface in $\mathbb{R}^{3}$ at a non-convex point, Analysis 5 (1985), 61-69. Corrigendum: Analysis 6 (1986), 413.

[31] K. Lancaster, Nonparametric minimal surfaces in $\mathbb{R}^{3}$ whose boundaries have a jump discontinuity, Inter. J. Math. Math. Sci. 11 (1988), 651-656.

[32] K. E. Lancaster, Existence and nonexistence of radial limits of minimal surfaces, Proc. Amer. Math. Soc. 106 (1989), 757-762. 
[33] K. E. Lancaster, Boundary behavior near reentrant corners for solutions of certain elliptic equations, Rend. Circ. Matem. Palermo (2) 40 (1991), 189-214.

[34] K. E. Lancaster, Notes on a talk given to the Wichita State Chapter of Pi Mu Epslion, Kirk Lancaster's personal files, Wichita State University, Wichita, KS, 2002.

[35] K. E. Lancaster, A Proof of the Concus Finn Conjecture, preprint.

[36] K. E. Lancaster and M. Athanassenas, CMC Capillary Surfaces at Reentrant Corners, Pacific J. Math. Vol. 234, No. 2 (2008), 201-228.

[37] K. E. Lancaster and D. Siegel, Existence and Behavior of the Radial Limits of a Bounded Capillary Surface at a Corner, Pacific J. Math. Vol. 176, No. 1 (1996), 165-194. Correction to figures, Pacific J. Math. Vol. 179, No. 2 (1997), 397-402.

[38] K.E. Lancaster and D. Siegel, Behavior of a bounded non-parametric H-surface near a reentrant corner, Z. Anal. Anwendungen 15 (1996), no. 4, 819-850.

[39] E. Miersemann, Asymptotic expansion at a corner for the capillary problem: the singular case, Pacific J. Math. 157 (1993), 95-107.

[40] D. E. Marshall and S. Rohde, Convergence of a Variant of the Zipper Algorithm for Conformal Mapping, SIAM J. on Numerical Analysis, 45 (2007), 2577-2609.

[41] D. E. Marshall, Lens chains and the geodesic algorithm for conformal mapping, preprint.

[42] C.P. Mitchell, An investigation of capillary surfaces at non-convex corners, Master's Thesis [532], Wichita State University, 2009.

[43] C.P. Mitchell, A Capillary Surface with No Radial Limits, submitted to the Pacific J. Math. preprint. arXiv:1702.01656

[44] V. N. Monakhov, Boundary-Value Problems with Free Boundaries for Elliptic Systems of Equations, Translations of Mathematical Monographs, AMS, Providence, 1980.

[45] Tristan Needham, Visual Complex Analysis, Oxford University Press, New York, 1997.

[46] Z. Nehari, Conformal Mapping, Dover Publications, New York,NY,1952.

[47] Francis Nguyen, Modules for the Investigation of the Central Fan Question through Numerical Computations, Wichita State University, Wichita, 2015.

[48] R. Osserman, A Survey of Minimal Surfaces, Dover, New York, 1986.

[49] J. Serrin, The problem of Dirichlet for quasilinear equations with many independent variables, Phil. Trans. Royal Soc. London Ser. A 264 (1969), 413-496.

[50] Danzhu Shi, Capillary Surfaces at a Re-entrant Corner, Pacific J. Math. 224 (2006), $321-353$. 
[51] Danzhu Shi \& R. Finn, On a theorem of Lancaster and Siegel, Pacific J. Math. 213 (2004), 111-119.

[52] D. Siegel, Height Estimates For Capillary Surfaces, Pacific J. Math. Vol.88, No. 2 (1980), 471-515.

[53] L. Simon, Regularity of capillary surfaces over domains with corners, Pacific J. Math. 88 (1980), 363-377.

[54] L. F. Tam, Regularity of capillary surfaces over domains with corners: borderline case, Pacific J. Math. 124 (1986), 469-482. 